\title{
Faecal Virome Analysis of Wild Animals from Brazil
}

\author{
Matheus A. Duarte ${ }^{1,2}$, João M. F. Silva ${ }^{2}{ }^{\circledR}$, Clara R. Brito ${ }^{1}$, Danilo S. Teixeira ${ }^{3}$, \\ Fernando L. Melo ${ }^{4}\left(\mathbb{D}\right.$, Bergmann M. Ribeiro ${ }^{2}\left(\mathbb{D}\right.$, Tatsuya Nagata ${ }^{2}$ and Fabrício S. Campos ${ }^{5, *(D)}$ \\ 1 Faculdade de Agronomia e Veterinária, Universidade de Brasília, Brasília-DF 70.910-900, Brazil \\ 2 Departamento de Biologia Celular, Instituto de Biologia, Universidade de Brasília, \\ Brasília-DF 70.910-900, Brazil \\ 3 Núcleo de Atendimento e Pesquisa de Animais Silvestres, Universidade Estadual de Santa Cruz, \\ Ilhéus-BA 45.662-900, Brazil \\ 4 Departamento de Fitopatologia, Instituto de Biologia, Universidade de Brasília, Brasília-DF 70.910-900, Brazil \\ 5 Laboratório de Bioinformática e Biotecnologia, Campus de Gurupi, Universidade Federal do Tocantins, \\ Tocantins-TO 77.410-570, Brazil \\ * Correspondence: camposvet@gmail.com
}

Received: 30 July 2019; Accepted: 28 August 2019; Published: 30 August 2019

\begin{abstract}
The Brazilian Cerrado fauna shows very wide diversity and can be a potential viral reservoir. Therefore, the animal's susceptibility to some virus can serve as early warning signs of potential human virus diseases. Moreover, the wild animal virome of this biome is unknown. Based on this scenario, high-throughput sequencing contributes a robust tool for the identification of known and unknown virus species in this environment. In the present study, faeces samples from cerrado birds (Psittacara leucophthalmus, Amazona aestiva, and Sicalis flaveola) and mammals (Didelphis albiventris, Sapajus libidinosus, and Galictis cuja) were collected at the Veterinary Hospital, University of Brasília. Viral nucleic acid was extracted, submitted to random amplification, and sequenced by Illumina HiSeq platform. The reads were de novo assembled, and the identities of the contigs were evaluated by Blastn and tblastx searches. Most viral contigs analyzed were closely related to bacteriophages. Novel archaeal viruses of the Smacoviridae family were detected. Moreover, sequences of members of Adenoviridae, Anelloviridae, Circoviridae, Caliciviridae, and Parvoviridae families were identified. Complete and nearly complete genomes of known anelloviruses, circoviruses, and parvoviruses were obtained, as well as putative novel species. We demonstrate that the metagenomics approach applied in this work was effective for identification of known and putative new viruses in faeces samples from Brazilian Cerrado fauna.
\end{abstract}

Keywords: Beak and feather disease virus (BFDV); chicken anemia virus (CAV); Adenoviridae; Psittacine adenovirus 3; Chapparvovirus; Gyrovirus; Norovirus; Smacoviridae

\section{Introduction}

Cerrado is a Brazilian savannah and one of the most diverse biomes in the world. However, it has been threatened by livestock and agricultural crop production expansion. This fact endangers not just local fauna but also adjacent biomes, such as the Amazon [1]. Associated with wildlife conservation, environmental degradation is a problem for public health, since wild animals can serve as reservoirs or intermediate hosts for new zoonotic pathogens such as viruses. Many zoonotic virus diseases have been emerging or re-emerging, especially, those caused by alphaviruses (Chikungunya virus, Mayaro virus, Madariaga virus) [2,3], bunyaviruses (Oropouche virus) [4], and flaviviruses (Zika, dengue, and yellow fever viruses) [5]. In this scenario, the deforestation can increase the contact between humans and animals, inclusively vectors, contributing to the emergence of diseases outbreaks in different regions of the world $[6,7]$. 
In Cerrado, the wildlife-livestock-human relationship is a reality [1]. Consequently, zoonoses surveillance is an important measure to control possible emerging diseases. In addition to livestock production, urban expansion brings another concern: the presence of pets, which includes exotic animals and domestic rodents, birds, and pigs. Local fauna has potential to become pathogen reservoirs, especially birds and rodents, that can spread zoonotic and non-zoonotic pathogens to these pets since their domestic counterparts are probably more susceptible to infection by these pathogens $[7,8]$. In addition, it is well reported the contribution of exotic animals to human diseases, with many examples of pathogens transmission, such as Salmonella enterica, Francisella tularensis, Chlamydophila psittaci, and Pasteurella multocida [8,9]. These animals can introduce new species or strains in nature and can harbor local isolates.

Most emerging diseases are zoonotic [10], so minimizing the contact between wild and non-wild animals is necessary. Epidemiological surveillance with focus in native fauna is a way to identify possible threats to humans and non-human animals that are hidden in reservoirs or that are as yet unknown. To achieve this goal, metagenomics is a powerful tool. It is estimated that there are approximately 1.67 million unknown viruses of key zoonotic viral families in mammal and bird hosts and that $631,000-827,000$ of them are potential zoonotic [11]. Considering this information and the occurrence of new zoonotic emerging diseases in Brazil, the aim of this study was to perform a virus metagenomic investigation to identify known and unknown viruses of faecal virome of birds and mammals of Brazilian Cerrado biome.

\section{Materials and Methods}

\subsection{Sample Collection}

Faecal samples from seven specimens of birds ( $n=4$ Amazona aestiva, $n=1$ Sicalis flaveola, and $n=2$ Psittacara leucophthalmus) and three specimens of mammals ( $n=1$ Didelphis albiventris, $n=1$ Sapajus libidinosus, and $n=1$ Galictis cuja) were collected early morning from the ground of the animal enclosures and individually placed in sterilized plastic recipients, in the Veterinary Hospital of the University of Brasilia in 2016. The animals showed clinical signs of apathy and were monitored. The samples were transported refrigerated to the Laboratory of Virology of the Cell Biology Department at University of Brasilia and stored in a freezer at $-80^{\circ} \mathrm{C}$.

\subsection{Viral Enrichment and Nucleic Acid Extraction}

Faecal samples were grouped in two pools-one (Pool 1) with only birds (1- A. aestiva and S. flaveola), and another (Pool 2) with mammals and birds (2-P. leucophthalmus, D. albiventris, S. libidinosus, and G. cuja). They were resuspended and homogenized vigorously in Hanks's balanced solution and centrifuged at $2500 \times \mathrm{g}$ for $90 \mathrm{~min}$ at $4{ }^{\circ} \mathrm{C}$. The supernatant was filtered using a $0.45 \mu \mathrm{m}$ syringe filter and ultracentrifugated on a $25 \%$ sucrose cushion at $190,000 \times \mathrm{g}$ for $4 \mathrm{~h}$ at $4{ }^{\circ} \mathrm{C}$. The pellets were resuspended in TE buffer (10 mM Tris pH 7.4; 1 mM EDTA pH 8.0) and treated with $100 \mathrm{U}$ of DNase I (Invitrogen, Carlsbad, EUA) and $20 \mathrm{U}$ of RNase A (Invitrogen, Carlsbad, EUA) at $37^{\circ} \mathrm{C}$ for $2 \mathrm{~h}$. The putative viral RNA and DNA present in the resulting sample were extracted using the commercial High Pure Viral Nucleic Acid Kit (Roche, Basel, Switzerland) following the manufacturer's instructions.

\subsection{Sequence-Independent Amplification of Viral Nucleic Acids}

Random PCRs were performed prior to the metagenomic sequencing using a particle-associated nucleic acid (PAN-PCR) approach [12]. For the extracted DNA, the first reaction was made in a final volume of $50 \mu \mathrm{L}$, containing $5 \mu \mathrm{L}(\sim 500 \mathrm{ng})$ of template, $0.8 \mu \mathrm{M}$ of the K-random-s primer ( $5^{\prime}$-GAC CAT CTA GCG ACC TCC ACM NN MNM-3'), $0.2 \mathrm{mM}$ of each dNTP, $1 \times$ PCR buffer, $2.5 \mathrm{mM} \mathrm{MgCl}_{2}$, and $1 \mathrm{U}$ of Taq DNA polymerase (Invitrogen, Carlsbad, USA). Amplification PCR condition was: initial denaturation cycle at $94{ }^{\circ} \mathrm{C}$ for $3 \mathrm{~min}$, followed by 35 cycles at $94{ }^{\circ} \mathrm{C}$ for $50 \mathrm{~s}, 53{ }^{\circ} \mathrm{C}$ for $50 \mathrm{~s}$, and $72{ }^{\circ} \mathrm{C}$ for $50 \mathrm{~s}$, and final extension at $72{ }^{\circ} \mathrm{C}$ for $3 \mathrm{~min}$. For generating products with the conserved region of the 
K-random-s primer, an extension reaction was performed using a Klenow fragment DNA polymerase (New England Biolabs, NEB, Ipswich, USA) with $20 \mu \mathrm{L}$ of template and $5 \mathrm{U}$ of enzyme at $37^{\circ} \mathrm{C}$ for $2 \mathrm{~h}$. A third reaction for the amplification of the products was done in a volume of $50 \mu \mathrm{L}$, containing $5 \mu \mathrm{L}$ of template, $0.4 \mu \mathrm{M}$ of the K-s primer (5'-GAC CAT CTA GCG ACC TCC AC-3'), $0.2 \mathrm{mM}$ of each dNTP, $1 \times$ PCR buffer, $2.5 \mathrm{mM} \mathrm{MgCl}_{2}$, and $1 \mathrm{U}$ of Taq DNA polymerase (Thermo-Fisher Scientific, Waltham, EUA). Amplification condition was the same as above. For the extracted RNA, a cDNA synthesis was carried out initially with $10 \mu \mathrm{L}$ of RNA sample and $2.5 \mu \mathrm{M}$ of the K-random-s primer incubated at $75^{\circ} \mathrm{C}$ for $5 \mathrm{~min}$, followed by a reaction with $200 \mathrm{U}$ of M-MuLV (NEB, Ipswich, USA), $40 \mathrm{U}$ of RNase OUT (Thermo-Fisher Scientific, Waltham, EUA), $0.05 \mathrm{M}$ of DTT, and $1 \times \mathrm{M}$-MuLV buffer incubated at $90^{\circ} \mathrm{C}$ for $10 \mathrm{~min}$ and at $42^{\circ} \mathrm{C}$ for $1 \mathrm{~h}$. Extension was also performed using a Klenow fragment DNA polymerase (NEB, Ipswich, USA) at the same conditions as for the DNA products. Final PCR was made in $50 \mu \mathrm{L}$ final reaction volume containing $5 \mu \mathrm{L}$ of cDNA, $0.4 \mu \mathrm{M}$ of the K-s primer, $0.2 \mathrm{mM}$ of each dNTP, $1 \times$ PCR buffer, $2.5 \mathrm{mM} \mathrm{MgCl}_{2}$, and $1 \mathrm{U}$ of Taq DNA polymerase (Thermo-Fisher Scientific, Waltham, EUA). The amplified products were visualized by electrophoresis in a $1 \%$ agarose gel and purified with the commercial Illustra GFX PCR DNA and Gel Band Purification Kit (SigmaAldrich, San Luis, USA) following the manufacturer's instructions.

\subsection{Metagenomic Sequencing and Bioinformatics}

The purified products were sheared and submitted to library construction using the TrueSeq DNA Nano kit at Macrogen Inc. (Seoul, South Korea). High-throughput sequencing was performed in Illumina HiSeq 2500 platform with 100 nt paired-end. Quality control of the reads was analyzed in FastQC software [13]. Trimming quality and filtering were carried out with BBDuk tool [14] with the removal of the adapters and primer sequences of right and left ends. The reads were de novo assembled in Megahit v1.1.3 [15] and in SPAdes 3.13.0 [16]. The kmer sizes specified were 21, 41, 61, 81, and 99 bases, and 21,33, 55, 77, and 99, respectively. The contigs were submitted to tblastx search against to the RefSeq Virus database of the NCBI with E-value cutoff of 1e-10. False positives were filtered using blastn search against to the non-redundant (nt) database with cutoff of 1e-20. Reads sequences were deposited in SRA database with the accession number PRJNA556823.

\subsection{Phylogenetic Analysis}

Eukaryotic viral sequences obtained in this work were deposited in GenBank with the following accession numbers-MN025529, MN025530, MN153802, MN175605, MN175606, MN175607, MN175608, MN175609, MN175610, MN175611, MN175612, MN175613, MN175614, and MN175615 - and used to posterior phylogenetic analysis. Phylogenetics analysis was performed for Adenoviridae, Anelloviridae, Circoviridae, Parvoviridae, and Smacoviridae families. Alignment was carried out using the MUSCLE and ClustalW algorithm, and the trees were constructed by Neighbor-Joining (NJ) and Maximum-likelihood (ML) methods in MEGA7 [17], RAxML v8.2 [18] and IQ-TREE v1.6.10 [19] software. The jModelTest v2.1.10 and ProtTest v3.0 tools were used to estimate the best substitution models. Bootstrap was performed with 1000 replicates.

\section{Results}

\subsection{Pool Information}

Samples were divided into two pools. Pool 1 was composed by faecal samples of A. aestiva and S. flaveola and pool 2 with P. leucophthalmus, D. albiventris, S. libidinosus, and G. cuja samples. A. aestiva is a psittacine species that has a wide distribution in Brazil and can be found in different natural habitats or as a pet. It occurs also in Argentina, Paraguay, and Bolivia. P. leucophthalmus, a psittacine species, extends widely over South America and is common in some urban areas [20]. S. flaveola is a passerine found naturally in South America [21]. D. albiventris is a marsupial found in Brazil, Paraguay, and Argentina, including urban areas [22]. S. libidinosus is a New World monkey 
endemic to Brazil that was recently found infected with Zika virus. It is found in Cerrado and Caatinga biomes [23,24]. G. cuja, a carnivore, is a mustelid with broad distribution over South America [25].

\subsection{Pool 1}

Illumina sequencing generated 22,586,752 and 25,049,662 paired-end reads for DNA and RNA samples, respectively. Reads were concatenated in a single archive. Final number of trimmed reads was 47,226,100. The contigs were de novo assembled using Megahit v1.1.3 and SPAdes 3.13.0 [15,16]. Megahit generated 22,986 contigs with average length of $813 \mathrm{nt}$ and standard deviation of 2043. Minimum and maximum contig lengths were $200 \mathrm{nt}$ and 222,414 nt. SPAdes produced 27,311 contigs with average length of $674 \mathrm{nt}$ and standard deviation of 1003. Minimum and maximum contig lengths were $100 \mathrm{nt}$ and 59,423 nt. Contigs of both assemblers were concatenated and submitted to tblastx search against RefSeq virus database and later to Blastn search against to the nt database. Contig with blast search hits with animal viruses is represented in Figures 1A and 2A,B. A list of eukaryotic viral contigs with significant tblastx hits and their GenBank accession numbers are shown in Table S1.

\subsection{Pool 2}

Illumina sequencing generated 22,992,728 and 28,478,188 paired-end reads for DNA and RNA samples. Reads were concatenated in a single archive. Final number of trimmed reads was 51,332,426. The contigs were de novo assembled using Megahit v1.1.3 and SPAdes 3.13.0 [15,16]. Megahit generated 2642 contigs with average length of $2113 \mathrm{nt}$ and standard deviation of 5683. Minimum and maximum contig lengths were $200 \mathrm{nt}$ and 80,761 nt. SPAdes produced 4139 contigs with average length of $1450 \mathrm{nt}$ and standard deviation of 3785. Minimum and maximum contig lengths were $100 \mathrm{nt}$ and 62,492 nt. The concatenated contigs were submitted to tblastx search against RefSeq virus database and later to Blastn search against to the Nucleotide database. Viral contigs classification is represented in Figures $1 \mathrm{~B}$ and 2C,D. A list of eukaryotic viral contigs with significant tblastx hits, and their GenBank accession numbers are shown in Table S2.

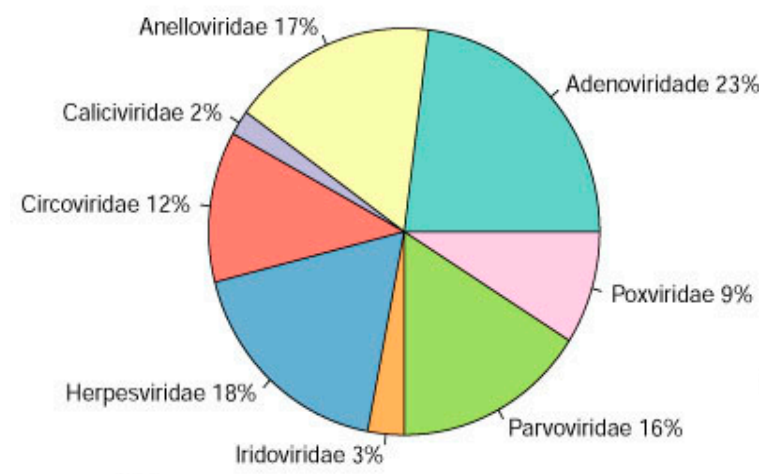

A)

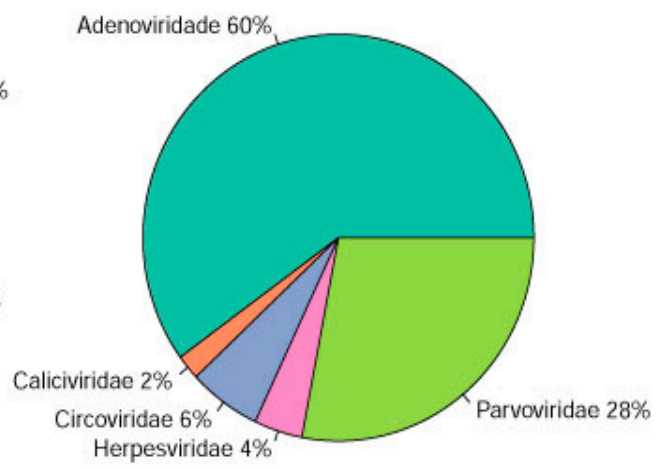

B)

Figure 1. Percentage of the contigs with blast search hits with animal viruses classified in families of pool 1 (A) and pool 2 (B) assembled using Megahit v1.1.3 and SPAdes 3.13.0 and filtrated by final tblastx. 
A)

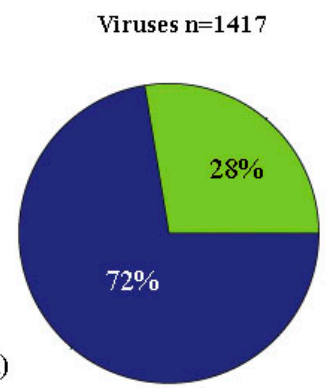

C)

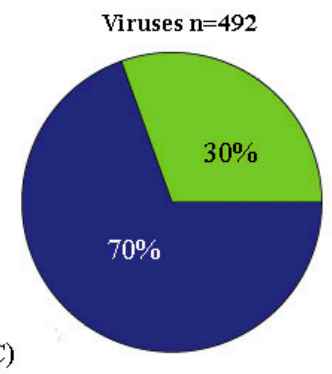

Viruses $n=1573$

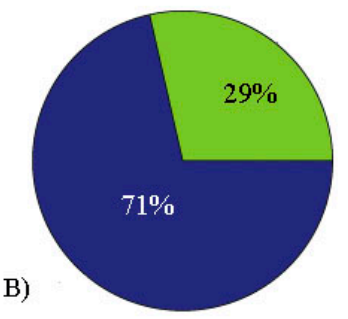

D)

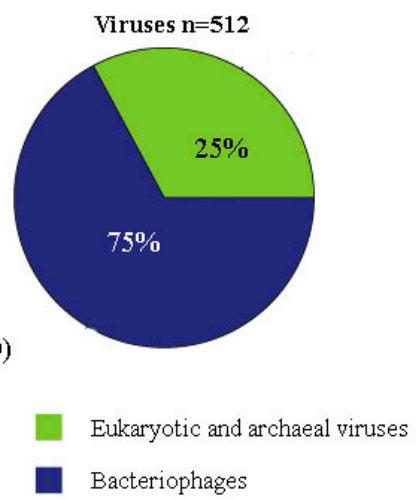

Figure 2. Viral contigs classification (bacteriophages, eukaryotic and archaeal viruses) represented by pie charts. A: contigs of pool 1 assembled using Megahit v1.1.3 and obtained by final tblastx filtration (cutoff: 10e-10). B: contigs of pool 1 assembled using SPAdes 3.13 .0 and obtained by final tblastx filtration (cutoff: 10e-10). C: contigs of pool 2 assembled using Megahit v1.1.3 and obtained by final tblastx filtration (cutoff: 10e-10). D: contigs of pool 2 assembled using SPAdes 3.13 .0 and obtained by final tblastx filtration (cutoff: 10e-10).

\subsection{Adenoviridae}

Adenoviridae is a family of non-enveloped dsDNA viruses with non-segmented linear genome of $26-48$ kilo-base pair ( $\mathrm{kb}$ or $\mathrm{kbp}$ ) in size. It is currently divided into five genera [26]. They are involved in many respiratory and gastrointestinal animal diseases and are included in surveillance programs given their importance in public health $[27,28]$. Adenovirus-like sequences close to Aviadenovirus and Atadenovirus genera were detected in pool 1. The same genera were detected in pool 2 besides Mastedonovirus. It is the viral family from both samples with the greatest number of viral contigs obtained. Amino acid identity ranges from $32.9 \%$ to $92.7 \%$ for pool 1, with contig length varying from 266 to $2606 \mathrm{nt}$, and $42.1 \%$ to $100 \%$ for pool 2, with contig length varying from 115 to $20,267 \mathrm{nt}$. Phylogenetic analyses were performed using DNA polymerase and hexon amino acid sequences of aviadenoviruses and atadenoviruses obtained from pool 2, including the most closely related sequences identified by tblastx search (Figures 3 and 4). For hexon amino acid sequence, pairwise identity between contig NODE 39 and Northern Aplomado falcon adenovirus (AAV90966.1) was 73.9\%. For contig k119 2350 and psittacine adenovirus 3 (Psittacine atadenovirus A) (YP_009112724.1), 97.6\%. For contig k119 1050 and Duck atadenovirus A (NP_044710.1), 64.5\%. DNA polymerase amino acid sequence pairwise shows identity of $90.2 \%$ between contig k119 2155 and psittacine adenovirus 3 (YP009112716.1). For contig k119 380 and Fowl aviadenovirus A (AP_000410.1), 61.7\% of amino acid identity. For contig k119 1050 and Duck atadenovirus A (NP_044710.1), 49.1\% (Table 1). Schematic genome representation of three novel putative adenovirus species is shown (Figure 5). 


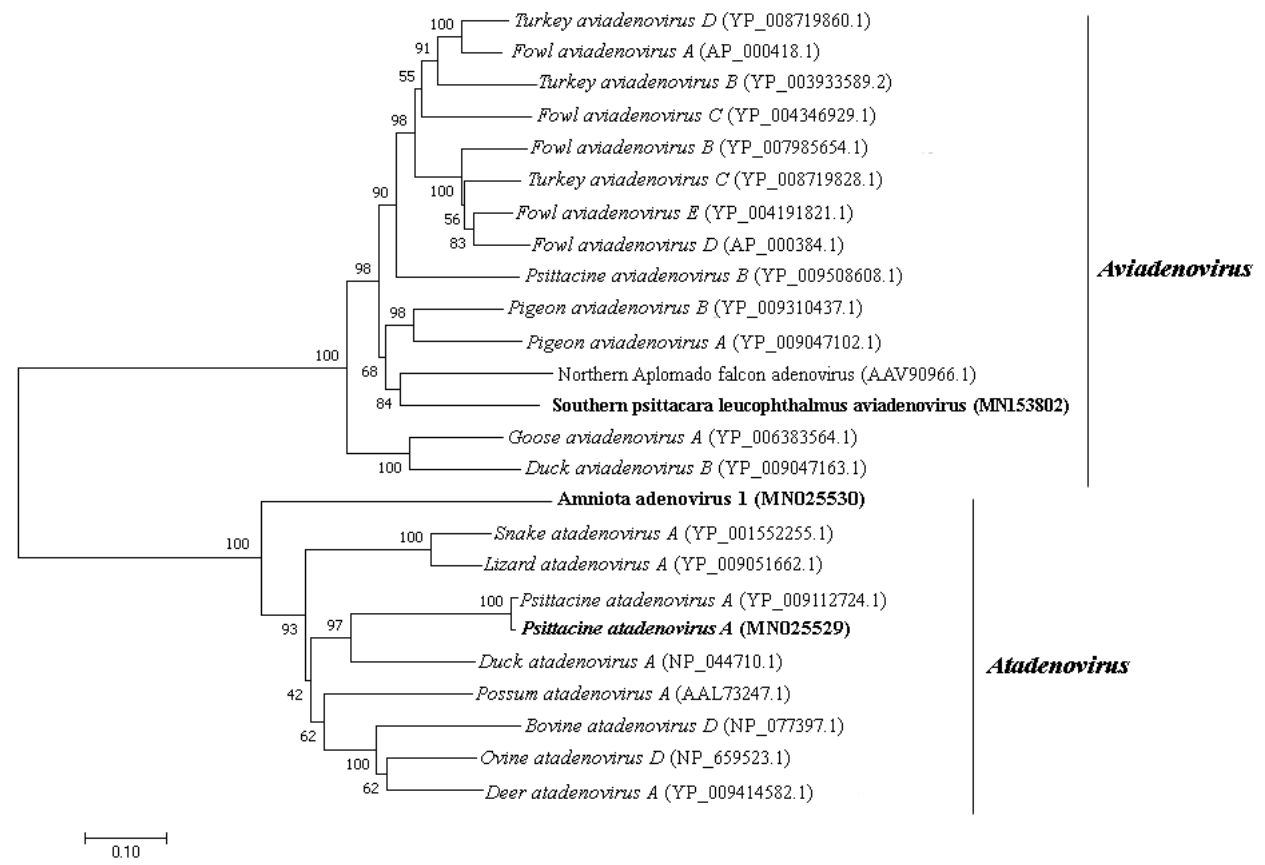

Figure 3. Neighbor-joining tree based on hexon protein amino acid sequence of ( 900 aa) 25 aviadenovirus and atadenovirus sequences. The tree is midpoint rooted and was built in MEGA7 software using Jones-Taylor-Thornton (JTT) substitution-rate matrix with gamma distribution $(+\mathrm{G})$ in accordance to ProtTest v3.0 analysis. Alpha shape parameter was estimated, and bootstrap was performed with 1000 replicates. Adenovirus sequences identified in this study are labeled in bold type. GenBank accession numbers of the viral sequences are shown in parentheses.

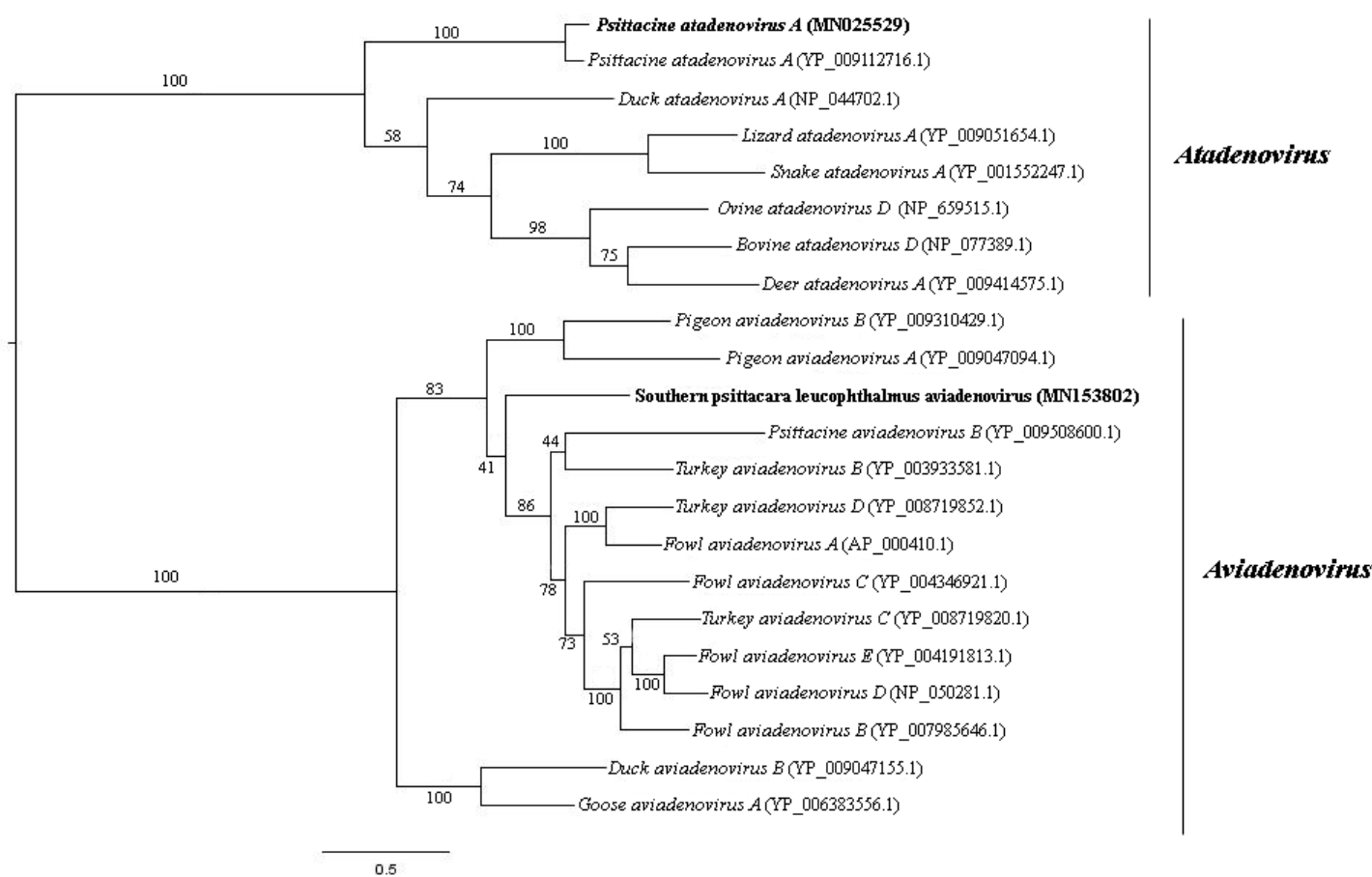

Figure 4. Maximum-likelihood tree based on DNA polymerase protein amino acid sequence ( 1300 aa) of 22 aviadenovirus and atadenovirus sequences. The tree is midpoint rooted and was built in RAxML v8.2 software using Le and Gascuel (LG) substitution-rate matrix with gamma distribution $(+G)$ and invariant sites $(+\mathrm{I})$ in accordance to ProtTest analysis. Bootstrap was performed with 1000 replicates. Adenovirus sequences identified in this study are labeled in bold type. GenBank accession numbers of the viral sequences are shown in parentheses. 
Table 1. Adenovirus-like contig ID identified from pool 2 and used for phylogenetic analyses with their respective pairwise identities.

\begin{tabular}{|c|c|c|c|c|c|c|}
\hline Pool Number & Contig ID & $\begin{array}{c}\text { GenBank } \\
\text { Accession Number }\end{array}$ & Virus Name & Closely Related Virus Type & $\begin{array}{l}\text { Pairwise Identity } \\
\text { (Hexon aa) }\end{array}$ & $\begin{array}{c}\text { Pairwise Identity } \\
\text { (DNA Polymerase aa) }\end{array}$ \\
\hline 2 & k119 1050 & MN025530 & Amniota adenovirus 1 & $\begin{array}{c}\text { Duck atadenovirus A } \\
\text { (NP_044710.1) }\end{array}$ & $64.5 \%$ & $41.9 \%$ \\
\hline 2 & k119 2350 & MN025529 & Psittacine adenovirus 3 & $\begin{array}{l}\text { Psittacine adenovirus } 3 \\
\quad \text { (YP_009112724.1) }\end{array}$ & $97.6 \%$ & - \\
\hline 2 & k119 2155 & MN025529 & Psittacine adenovirus 3 & $\begin{array}{l}\text { Psittacine adenovirus } 3 \\
\quad \text { (YP009112716.1) }\end{array}$ & 一 & $90.2 \%$ \\
\hline 2 & k119 380 & MN153802 & $\begin{array}{l}\text { Southern psittacara } \\
\text { leucophthalmus } \\
\text { aviadenovirus }\end{array}$ & $\begin{array}{l}\text { Fowl aviadenovirus } A \\
\quad\left(\mathrm{AP} \_000410.1\right)\end{array}$ & - & $61.7 \%$ \\
\hline 2 & NODE 39 & MN153802 & $\begin{array}{l}\text { Southern psittacara } \\
\text { leucophthalmus } \\
\text { aviadenovirus }\end{array}$ & $\begin{array}{l}\text { Northern Aplomado falcon } \\
\text { adenovirus (AAV90966.1) }\end{array}$ & $73.9 \%$ & - \\
\hline
\end{tabular}


A)

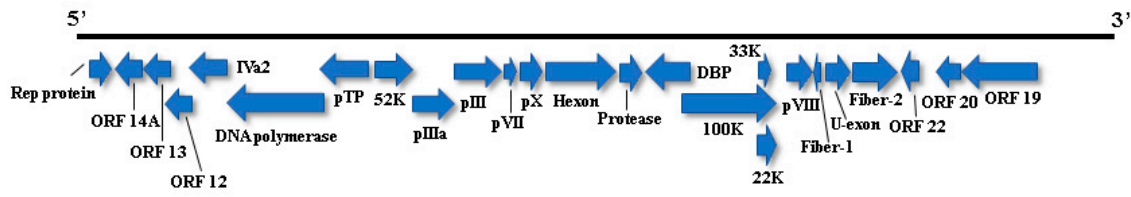

B)

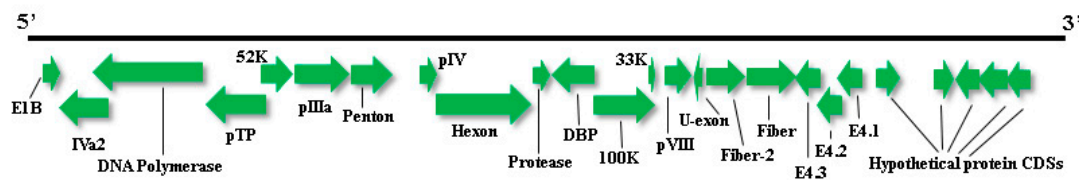

C)

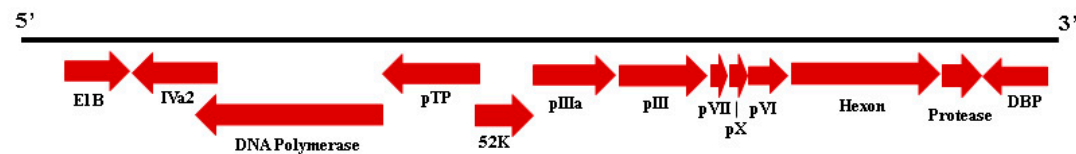

Figure 5. Schematic genome representation of three adenoviruses. A: in blue, a possible new adenovirus, the proposed southern psittacara leucophthalmus aviadenovirus with nearly complete genome obtained $(\sim 35 \mathrm{~kb})$. B: in green, nearly complete viral genome sequence of the proposed psittacine adenovirus 3 isolate BR_DF $(\sim 30 \mathrm{~kb})$. C: in red, a putative new species with partial genome, the proposed amniota adenovirus $1(\sim 18 \mathrm{~kb})$.

\subsection{Anelloviridae}

Anelloviruses are non-enveloped viruses with negative sense and circular ssDNA genome with $2.1-3.9 \mathrm{~kb}$ in size. They have a wide distribution in human population and were found in different vertebrate species, including birds and mammals [29]. Anellovirus-like sequences are present only in pool 1. Most contigs were related to ORF1 of Seal anellovirus 4 after tblastx search. Their amino acid identity varied from $33.3 \%$ to $52.5 \%$ and sequence length from 425 to $2659 \mathrm{nt}$. Contig sequences closer to giant panda anellovirus, Torque teno canis virus and Torque teno sus virus $k 2 b$, showed amino acid identities of $34.4 \%, 36.0 \%$ to $39.8 \%$, and $52.5 \%$, with lengths of $882 \mathrm{nt}, 962$ to $1250 \mathrm{nt}$, and $555 \mathrm{nt}$, respectively. Chicken anemia virus, avian gyrovirus 2, and gyrovirus GyV3 species were also detected (Figure 6A). ORF1 nucleotide sequence was used for phylogenetic analyses since it is used as species demarcation criteria (Figures 7 and 8). Between chicken anemia virus isolate (contig k199 16753) and closely related isolate strain CL37 (JQ308213.1), nucleotide identity was 98.8\%. Nucleotide identity between contig k119 6992 and most closely related avian gyrovirus 2 isolate (KX708510.1) was 98.8\%. Between contig k119 6843 and most closely related gyrovirus GyV3 (MG366592.1) was 99.4\%. For contigs NODE 177, NODE 986 and NODE 1090, identity with giant panda anellovirus (MF327552.1) was 54.6\%, 50.7\% and 51.7\% respectively (Figure 6B) (Table 2). A phylogenetic tree was constructed including just gyroviruses sequences. Another phylogenetic tree was built with the main genera of Anelloviridae family and unclassified closely related anelloviruses. 


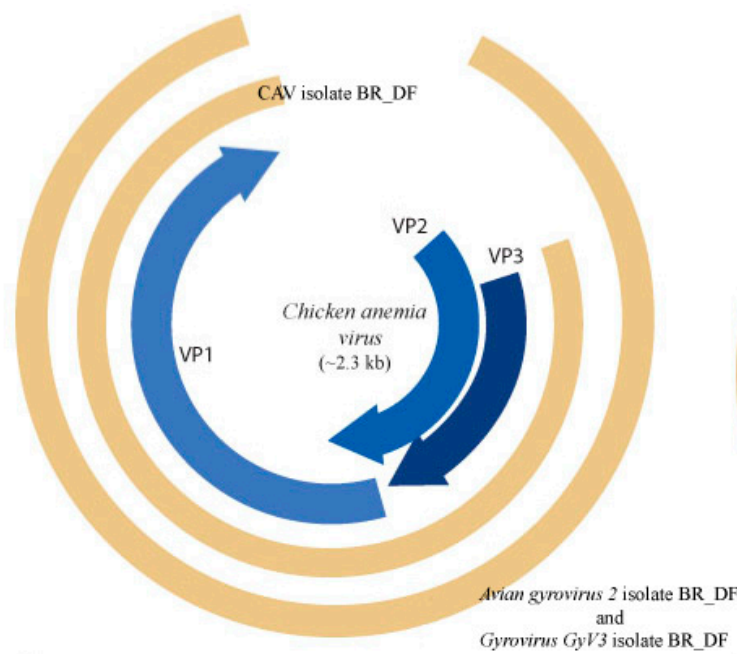

A)

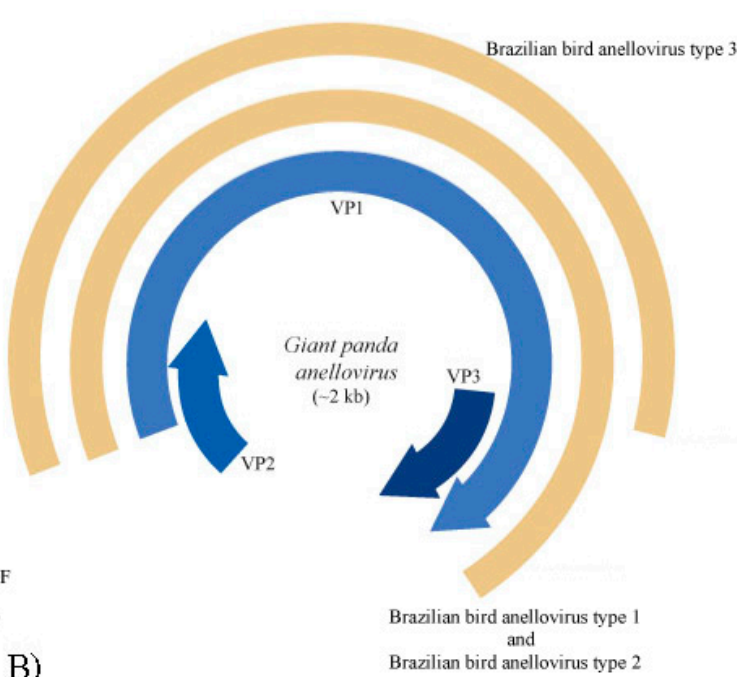

Brazilian bird and anellovirus type 2

Figure 6. Genome representation of known and novel anelloviruses. A: In blue, ORFs VP1, VP2 and VP3 of the prototypic gyrovirus, chicken anemia virus (CAV), and, in brown, the proposed CAV isolate BR_DF (contig k199 16753), avian gyrovirus 2 isolate BR_DF (contig k119 6992) and gyrovirus GyV3 isolate BR_DF (contig k119 6843). B: In brown, the proposed Brazilian bird anellovirus type 1, type 2 and type 3 (contigs NODE 177, NODE 986 and NODE 1090, respectively) and, in blue, ORFs VP1, VP2, and VP3 of the closely related giant panda anellovirus.

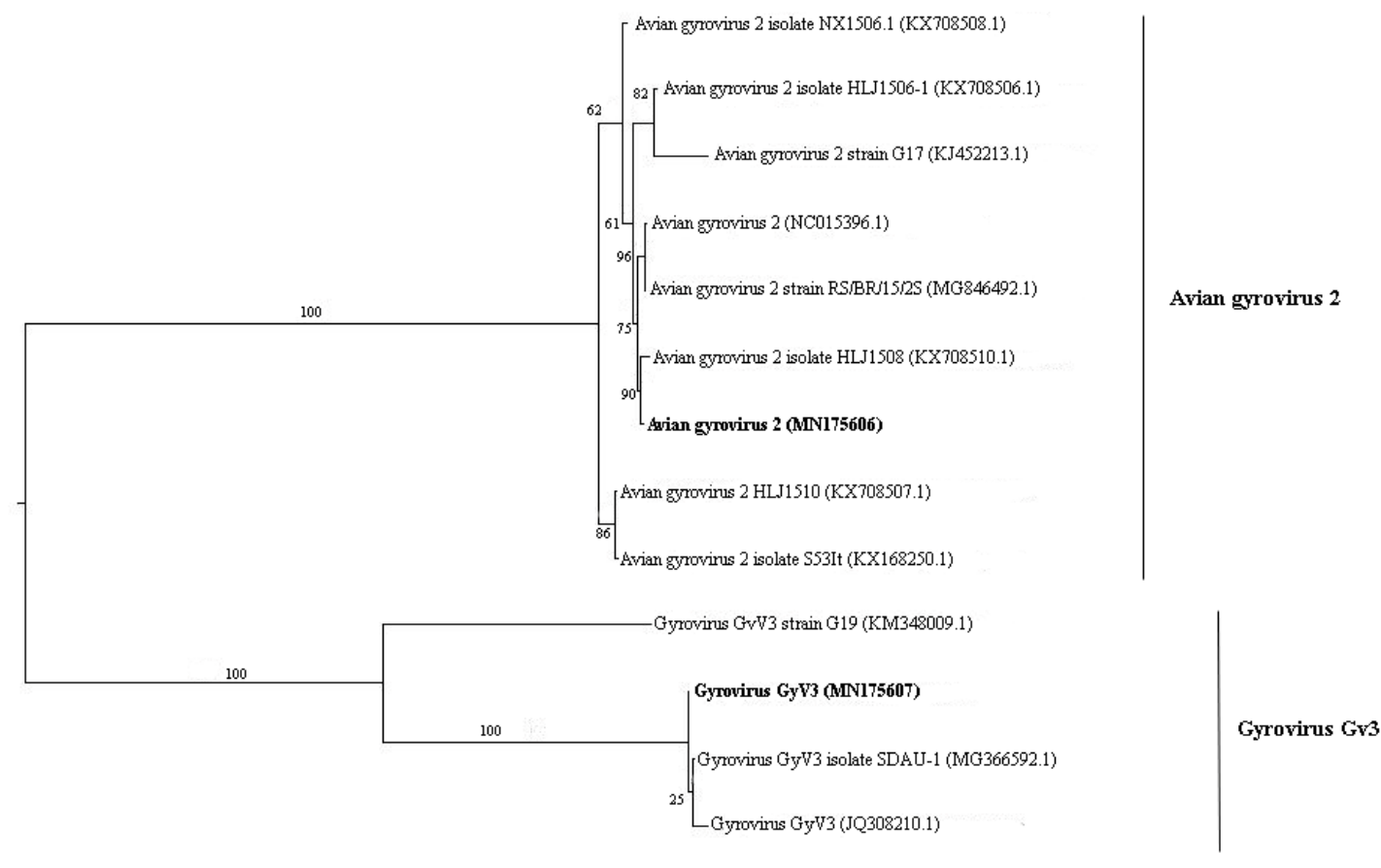

Figure 7. Maximum-likelihood tree based on ORF 1 nucleotide sequence ( 1.4 kb) of 13 avian gyrovirus 2 and gyrovirus GyV3 sequences. The tree is midpoint rooted and was built in RAxML v8.2 software using general time-reversible (GTR) substitution model with gamma distribution $(+\mathrm{G})$ in accordance to jModelTest v2.1.10 analysis. Bootstrap was performed with 1000 replicates. Anellovirus sequences identified in this study are labeled in bold type. GenBank accession numbers of the viral sequences are shown in parentheses. 


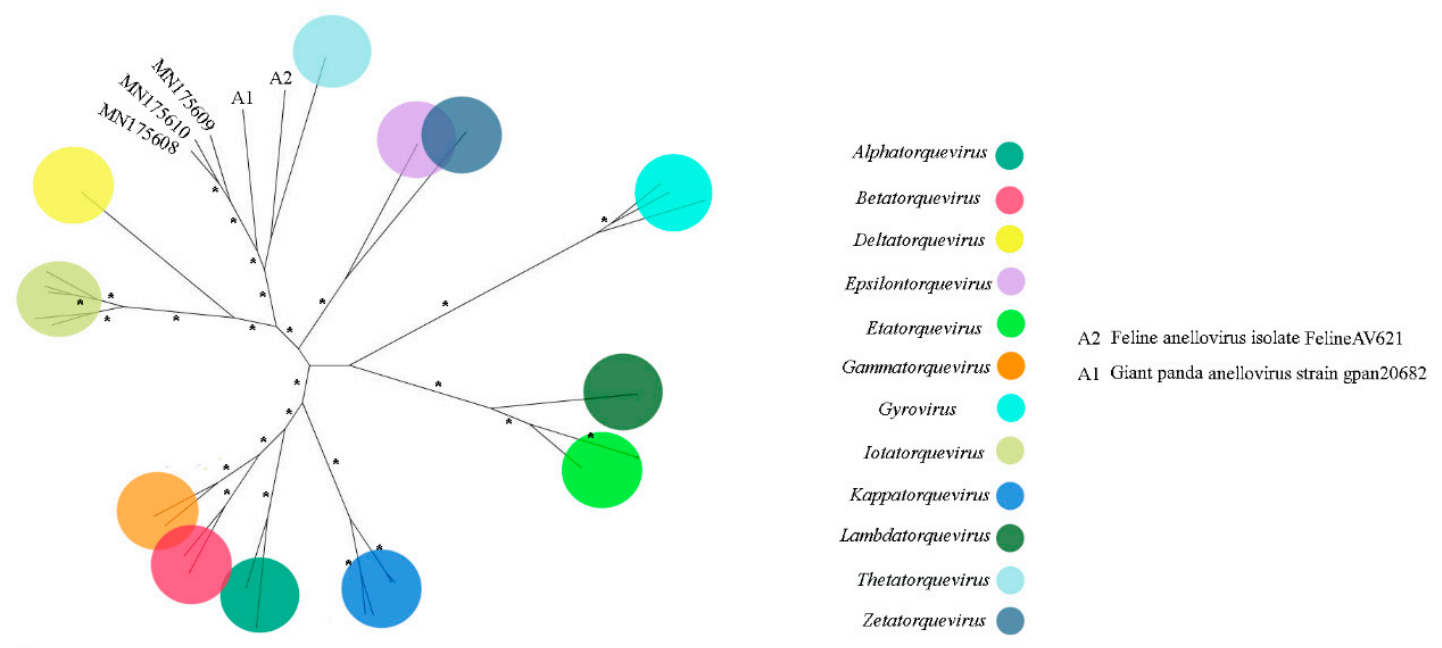

Figure 8. Maximum-likelihood tree based on ORF 1 nucleotide sequence $(\sim 2.3 \mathrm{~kb})$ of 32 anellovirus. The tree is unrooted and was built in IQ-TREE v1.6.10 software using transversion substitution model (TVM) with gamma distribution $(+\mathrm{G})$ and invariant sites $(+\mathrm{I})$ in accordance to jModelTest v2.1.10 analysis. Bootstrap was performed with 1000 replicates, and values equal to 70 or more are represented by asterisks. GenBank accession numbers of the anellovirus sequences identified in this study are shown. Details about the sequences of the phylogenetic tree are shown in Table S3.

Table 2. Anellovirus-like contig ID identified from pool 1 and used for phylogenetic analyses with their respective pairwise identities.

\begin{tabular}{|c|c|c|c|c|c|}
\hline Pool Number & Contig ID & $\begin{array}{c}\text { GenBank } \\
\text { Accession Number }\end{array}$ & Virus Name & $\begin{array}{c}\text { Closely Related Virus } \\
\text { Type }\end{array}$ & $\begin{array}{l}\text { Pairwise Identity } \\
\text { (ORF nt) }\end{array}$ \\
\hline 1 & k119 16753 & MN175605 & $\begin{array}{l}\text { Chicken anemia } \\
\text { virus }\end{array}$ & $\begin{array}{l}\text { Chicken anemia virus } \\
\text { (JQ308213.1) }\end{array}$ & $98.8 \%$ \\
\hline 1 & k119 6992 & MN175606 & Avian gyrovirus 2 & $\begin{array}{c}\text { Avian gyrovirus } 2 \\
\text { (KX708510.1) }\end{array}$ & $98.8 \%$ \\
\hline 1 & k119 6843 & MN175607 & Gyrovirus GyV3 & $\begin{array}{c}\text { Gyrovirus GyV3 } \\
\text { (MG366592.1) }\end{array}$ & $99.4 \%$ \\
\hline 1 & NODE 177 & MN175608 & $\begin{array}{l}\text { Brazilian bird } \\
\text { anellovirus type } 1\end{array}$ & $\begin{array}{c}\text { Giant panda anellovirus } \\
\text { (MF327552.1) }\end{array}$ & $54.6 \%$ \\
\hline 1 & NODE 986 & MN175609 & $\begin{array}{c}\text { Brazilian bird } \\
\text { anellovirus type } 2\end{array}$ & $\begin{array}{l}\text { Giant panda anellovirus } \\
\text { (MF327552.1) }\end{array}$ & $50.7 \%$ \\
\hline 1 & NODE 1090 & MN175610 & $\begin{array}{l}\text { Brazilian bird } \\
\text { anellovirus type } 3\end{array}$ & $\begin{array}{c}\text { Giant panda anellovirus } \\
\text { (MF327552.1) }\end{array}$ & $51.7 \%$ \\
\hline
\end{tabular}

\subsection{Caliciviridae}

Caliciviridae is a viral family composed of 11 genera of small non-enveloped viruses with non-segmented, linear, positive-sense ssRNA genome that ranges in size from 7.3-8.3 kb. Important animal pathogens that cause enteric and respiratory diseases are included in this family [30,31]. Few calicivirus-like sequences were identified in pools 1 and 2 . All the contigs were closely related to Norovirus genus, specifically to norovirus GII and GI for pool 1 and norovirus GI for pool 2. Amino acid identity ranged from $70.1 \%$ to $96.3 \%$ and $79.7 \%$ to $80.3 \%$, with small contigs length of 419 to 501 and 441 to $447 \mathrm{nt}$, respectively (Figure 9). VP1 and VP2 amino acid sequences were not included in the phylogenetic analyses given the small contig length obtained. 


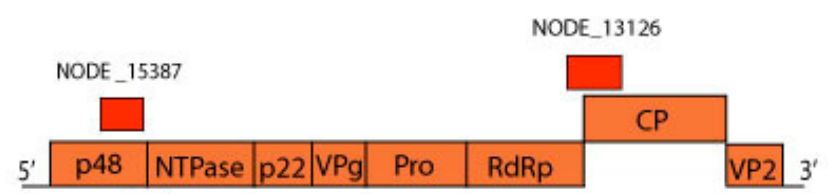

Figure 9. Norovirus genome representation with contigs NODE 15387 (MN175617) and NODE 13126 (MN175616) of pool 1 aligned to ORF1 and ORF2, that encode a polyprotein and capsid protein, respectively.

\subsection{Circoviridae}

Recently submitted to taxonomic revision, circoviruses are small, non-enveloped viruses with circular ssDNA genome ranging between $1.7-2.3 \mathrm{~kb}$ in size that belong to the circular rep-encoding single-strand (CRESS) DNA virus group [32]. Vertebrate and invertebrate hosts have been described for these viruses, affecting especially avian and swine with the smallest known animal viral pathogens included in this group [33,34]. Circovirus-like sequences were detected in both pools and are closely related to Circovirus genus, specifically to beak and feather disease virus (BFDV). Partial and complete genome sequences were obtained. Amino acid identity ranged from $73.1 \%$ to $96.5 \%$ and contigs length between 308 and $1999 \mathrm{nt}$. Phylogenetic analyses were performed considering genome-wide pairwise identities as demarcation threshold in the group. Nucleotide identity between the isolate BR_DF (contig k199 22721), from the present study, and closest isolate, BFDV-U_PL-543_2008 (JX221029.1), was $94.9 \%$ (Figure 10).

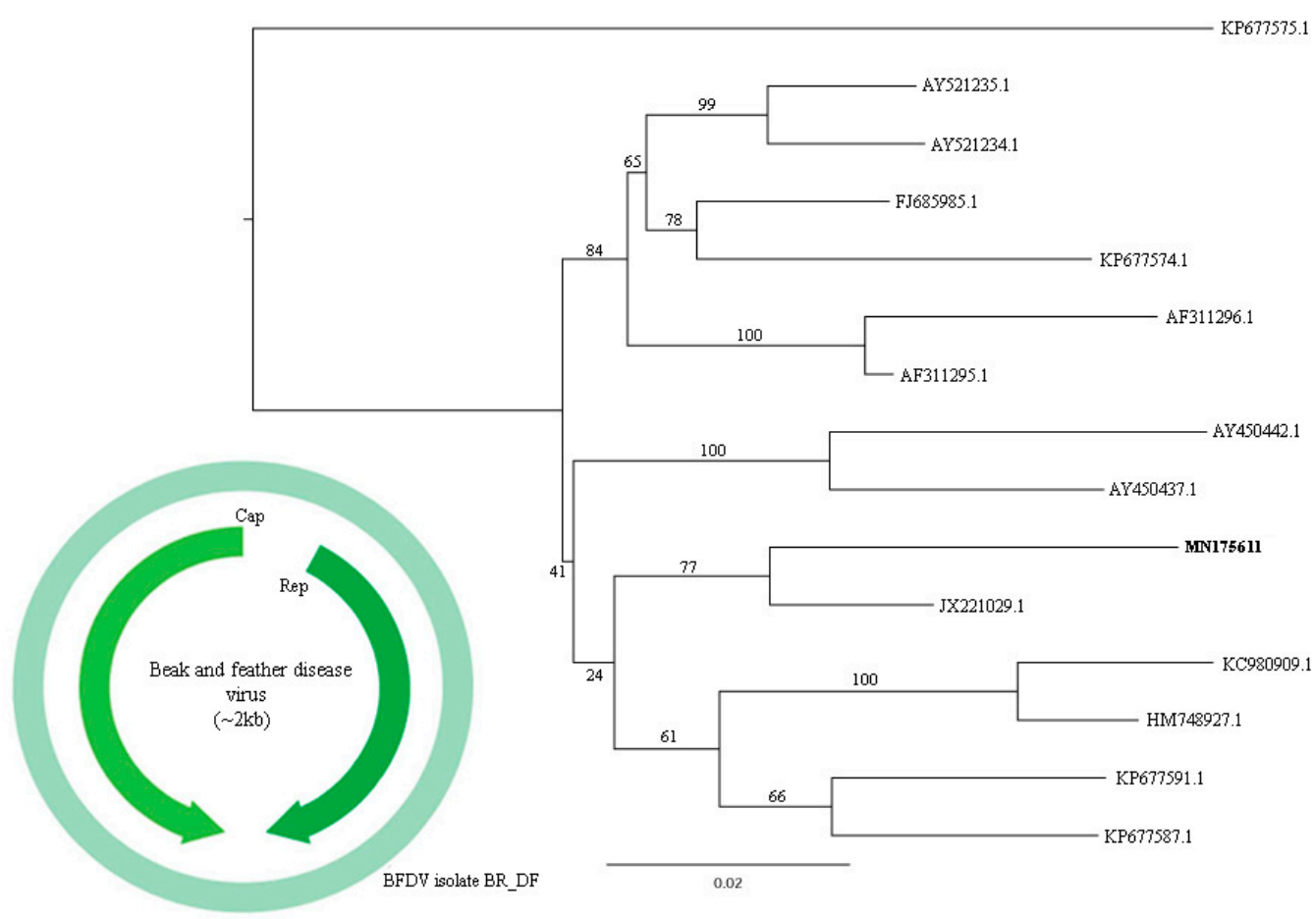

Figure 10. Maximum-likelihood tree based on whole genome nucleotide sequence ( 2 kb) of 15 BFDV sequences. The tree is midpoint rooted and was built in IQ-TREE v1.6.10 software using Tamura-Nei nucleotide substitution model $(\operatorname{TrN})$ with gamma distribution $(+\mathrm{G})$ and invariant sites $(+\mathrm{I})$ in accordance to jModelTest v2.1.10 analysis. Bootstrap was performed with 1000 replicates. Circovirus sequence identified in this study are labeled in bold type. GenBank accession numbers of the viral sequences are shown. In light green, the first complete genome sequence of a BFDV Brazilian isolate (MN175611), with capsid and replication proteins represented. 


\subsection{Parvoviridae}

Parvoviridae is a viral family of small non-enveloped ssDNA viruses with non-segmented and linear genome of $4-6.3 \mathrm{~kb}$ in size, involved in many clinical and subclinical animal infections. It is divided into subfamilies Densovirinae, found infecting arthropods, and Parvovirinae, that infect vertebrates $[35,36]$. For pool 1, the contigs length ranged from 319 to $4116 \mathrm{nt}$, showing amino acid identity from $28.9 \%$ to $84.5 \%$ with other parvoviruses. For pool 2, length varied from 120 to $4425 \mathrm{nt}$ and amino acid identity from $44.0 \%$ to $84.8 \%$. Viruses related to both subfamilies were present in pool 1 and are closely related to Ambidensovirus, Iteradensovirus, Dependoparvovirus, and Chapparvovirus genera. For pool 2, just viruses closely related to Parvovirinae (Dependoparvovirus and Chapparvovirus) were detected. Three nearly complete genome sequences were obtained (Figure 11). The conserved NS1 protein amino acid sequence is a demarcation criterion for the group and was used for phylogenetic analyses (Figure 12). The contigs k119 1463 and k119 15398 showed amino acid identity of 45.3\% and 44.9\% to turkey parvovirus TP1-2012/HUN (AHF54687.1), respectively. The contig k119 1997 and adeno-associated virus (YP_009552823.1) showed 42.7\% amino acid identity. On the other hand, NS1 amino acid identity between k199 1463 and k119 15398 was 56.0\% (Table 3).

A)

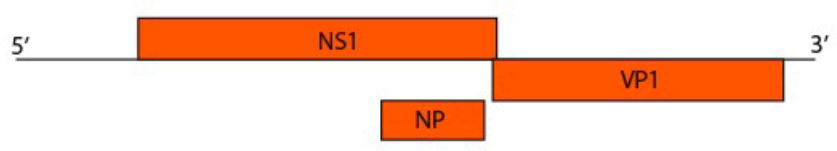

B)

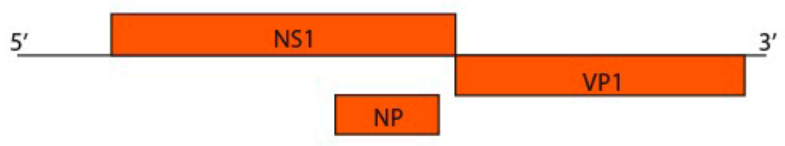

C)

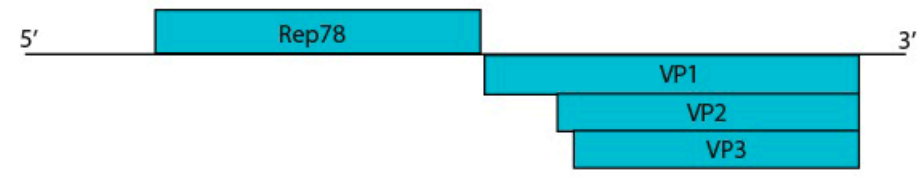

Figure 11. Genome representation of three putative novel parvoviruses identified. A: Proposed avian chapparvovirus (contig k119 1463) with conserved NS1 and VP1 sequences, and putative NP (4425 nt). B: Proposed psittacara leucophthalmus chapparvovirus (contig k119 15398) species (4116 nt). C: Proposed avian adeno-associated virus isolate BR_DF (contig k119 1997) with non- and structural protein sequences (4642 $\mathrm{nt})$. 

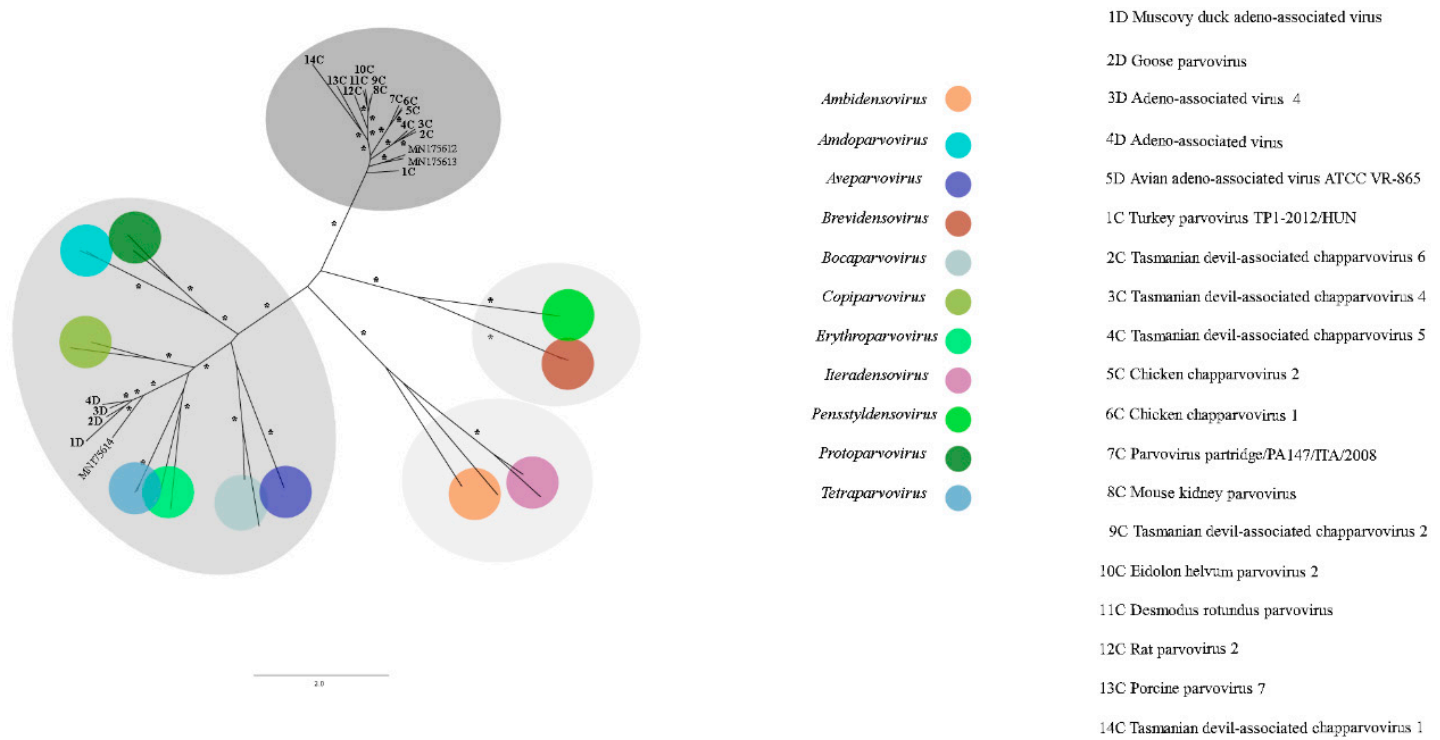

Figure 12. Maximum-likelihood tree based on non-structural protein 1 (NS1) amino acid sequence ( 800 aa) of 44 parvoviruses. The tree is unrooted and was built in IQ-TREE v1.6.10 software using rtREV substitution-rate matrix with gamma distribution $(+\mathrm{G})$, invariant sites $(+\mathrm{I})$ and empirical amino acid frequency $(+\mathrm{F})$ in accordance to ProtTest analysis. Bootstrap was performed with 1000 replicates and values equal to 70 or more are represented by asterisks. Chapparvoviruses are in darker grey. Densovirinae and Parvovirinae subfamilies in light and less dark grey respectively. GenBank accession numbers of the parvovirus sequences identified in this study are shown. Details about the sequences of the phylogenetic tree are shown in Table S3.

Table 3. Parvovirus-like contig ID identified from pool 1 and 2 used for phylogenetic analyses with their respective pairwise identities.

\begin{tabular}{|c|c|c|c|c|c|}
\hline Pool Number & Contig ID & $\begin{array}{c}\text { GenBank } \\
\text { Accession Number }\end{array}$ & Virus Name & $\begin{array}{c}\text { Closely Related Virus } \\
\text { Type } \\
\end{array}$ & $\begin{array}{l}\text { Pairwise Identity } \\
\text { (NS1 aa) }\end{array}$ \\
\hline 1 & k119 1463 & MN175612 & $\begin{array}{c}\text { Avian } \\
\text { chapparvovirus }\end{array}$ & $\begin{array}{l}\text { Turkey parvovirus } \\
\text { TP1-2012/HUN } \\
\text { (AHF54687.1) }\end{array}$ & $45.3 \%$ \\
\hline 2 & k119 15398 & MN175613 & $\begin{array}{c}\text { Psittacara } \\
\text { leucophthalmus } \\
\text { chapparvovirus }\end{array}$ & $\begin{array}{c}\text { Turkey parvovirus } \\
\text { TP1-2012/HUN } \\
\text { (AHF54687.1) }\end{array}$ & $44.9 \%$ \\
\hline 1 & k119 1997 & MN175614 & $\begin{array}{c}\text { Avian } \\
\text { adeno-associated } \\
\text { virus isolate BR_DF }\end{array}$ & $\begin{array}{l}\text { Adeno-associated virus } \\
\text { (YP_009552823.1)) }\end{array}$ & $42.70 \%$ \\
\hline
\end{tabular}

\subsection{Smacoviridae}

Accepted very recently by ICTV, smacoviruses are a group of CRESS viruses with genomes ranging from $2.3-2.9 \mathrm{~kb}$. They were identified by metagenomics in vertebrate faeces and insects and, so far, are not related to any animal disease. At present, the family Smacoviridae is divided into six genera [37]. Smacovirus-like sequences were found just in pool 1. All of them were closely related to Porprismacovirus genus, in which possible hosts include mammals and birds. Contigs length varied from 620 to $3091 \mathrm{nt}$ and replication-associated protein (rep) amino acid identity with other smacoviruses ranged from $57.8 \%$ to $90.3 \%$. Genome-wide identity between contig NODE 726 (MN175615) and the closest smacovirus, Lemur associated porprismacovirus 1 isolate SF5 (NC_026320.1), was 67.3\% (Figure 13). Phylogenetic analyses were performed using genome-wide and rep amino acid sequence, since the capsid protein $(\mathrm{CP})$ and the replication protein have different evolutionary histories due recombination in the family (Figure 14). 


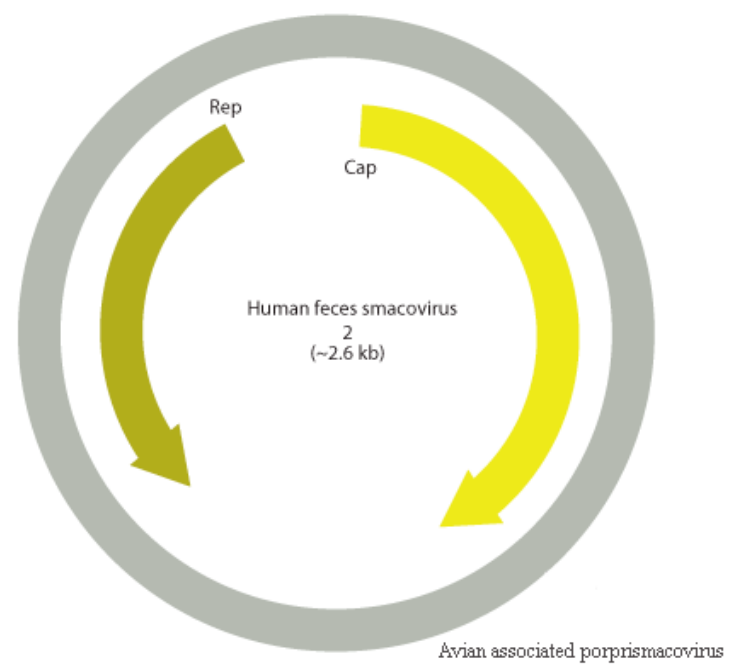

Figure 13. Novel smacovirus, the proposed Avian associated porprismacovirus (MN175615), represented in grey with Human feces smacovirus 2 as prototype for comparison.
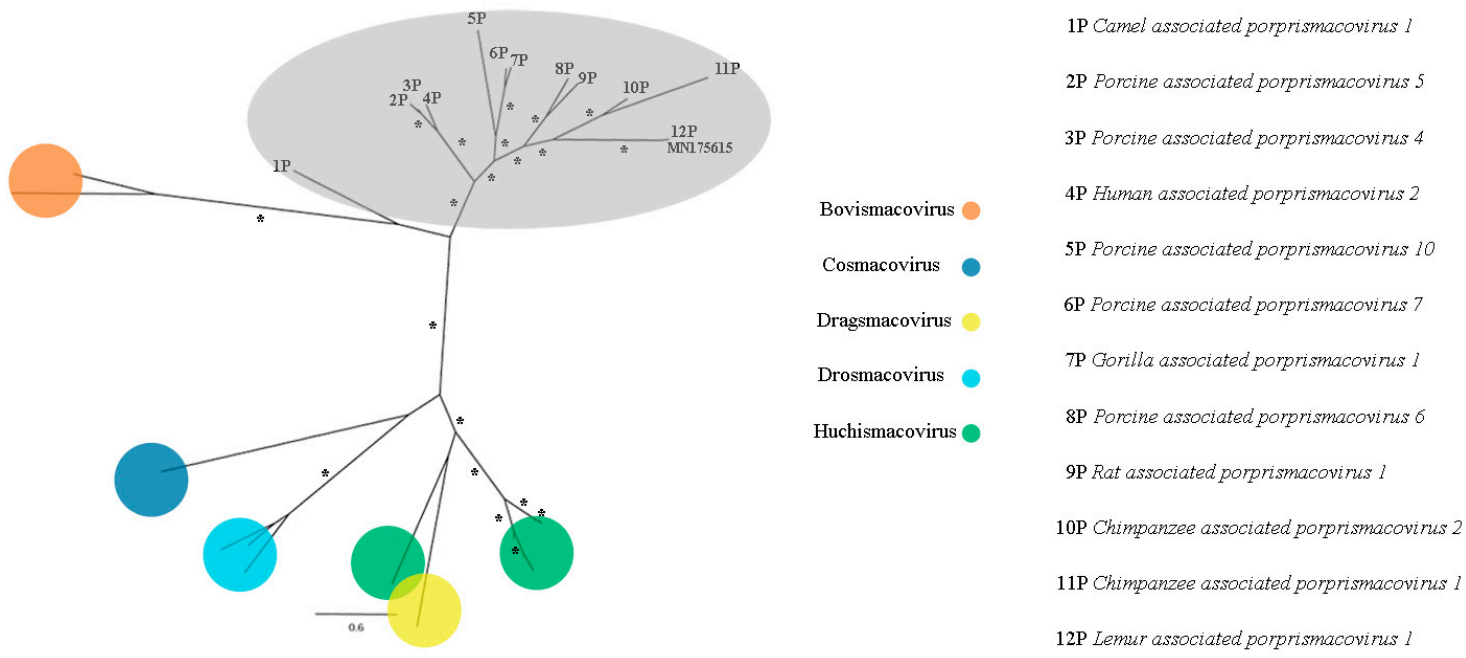

Figure 14. Maximum-likelihood tree based on replication-associated protein (rep) amino acid sequence ( $306 \mathrm{aa})$ of 28 smacovirus sequences. The tree is midpoint rooted and was built in RAxML v8.2 software using Le and Gascuel (LG) substitution-rate matrix with gamma distribution $(+G)$, invariant sites $(+\mathrm{I})$, and empirical amino acid frequency $(+\mathrm{F})$ in accordance to ProtTest analysis. Bootstrap was performed with 1,000 replicates and values equal to 70 or more are represented by asterisks. Nodes in grey area belongs to Porprismacovirus genus. GenBank accession number of the proposed Avian associated porprismacovirus identified in this study is shown. Details about the sequences of the phylogenetic tree are shown in Table S3.

\section{Discussion}

We applied a high-throughput sequencing method to investigate the faecal virome of specimens of wild animals of Cerrado biome, birds (Amazona aestiva, Psittacara leucophthalmus, and Sicalis flaveola) and mammals (Didelphis albiventris, Sapajus libidinosus, and Galictis cuja). Birds are considered important reservoir hosts of emerging viruses. At least, new 73 viruses were discovered between 2012 and 2014 in this group of animals [38]. Until 2017, these novel described viruses were documented mainly in wild birds, with Poxviridae, Herpesviridae, and Adenoviridae as the most reported DNA virus families with veterinary importance [39]. Regarding to mammals, many investigations focus mainly on bats species [40,41]. Canids [42] and rodents [43] are other groups commonly investigated. In the present study, members of Adenoviridae, Anelloviridae, Circoviridae, Caliciviridae, and Parvoviridae families were 
identified. Aviadenovirus and Atadenovirus sequences were detected in pool 2. Phylogenetic distance of DNA polymerase amino acid sequences greater than $5-15 \%$ is one of the several species demarcation criteria in these genera [44]. Considering those criteria, some of our newly identified viruses may merit establishing a novel species for them. By phylogenetic analyses, contig k199 2155 was grouped with psittacine adenovirus 3 (Psittacine atadenovirus A) (Figure 4), showing 90.2\% of amino acid identity. Hexon sequence in contig k119 2350 also showed high amino acid identity (97.2\%) with psittacine adenovirus 3 and was also grouped together (Figure 3). Therefore, these sequences, contigs k199 2155 and k199 2350, represent a new isolate of this virus, with nearly complete genome from P. leucophthalmus (Figure 5B). The first description of psittacine adenovirus 3 was in 2014 in an outbreak of avian chlamydiosis and human psittacosis in Hong Kong. In this occasion, it was supposed that this adenovirus caused an immunosuppression that favored Chlamydophila psittaci infection in Amazona farinosa parrots, resulting in transmission to humans [45]. No other identification was documented since then.

Low amino acid identity of one hexon sequence in contig k119 1050 indicates a putative novel adenovirus species more closely related to duck adenovirus 1 (DAdV-1) (Figures 3 and 5C), the proposed amniota adenovirus 1 . DAdV-1, is the etiologic agent of egg drop syndrome in gallinaceous birds, a disease of great economic importance [46]. DNA polymerase amino acid pairwise identity of contig k119 380 with the closest adenovirus identified, Fowl aviadenovirus A (FAdV-1) strain CELO, suggests the presence of a novel species in P. leucophthalmus that we tentatively named southern psittacara leucophthalmus adenovirus. Probably this species is closer to Falcon adenovirus 1 (FaAdV-1) analyzing hexon amino acid sequence (contig NODE 39), however no DNA polymerase sequence of FaAdV-1 is available. This virus, first detected in Falco femoralis septentrionalis, is involved in severe infectious disease in falcons, characterized by hepatitis, splenomegaly, and enteritis [47]. Further investigation is necessary to evaluate pathogenic potential of this new virus $[48,49]$.

Anellovirus-like sequences were also detected. Species and genus demarcation criteria in Anelloviridae are based on ORF1 nucleotide sequence identity with cut-off values, respectively, of the $35 \%$ and $56 \%$ [50]. Three known anellovirus species were detected in pool 1, specifically belonging to the genus Gyrovirus. This genus was recently reassigned from Circoviridae to Anelloviridae considering genomic features of this group [51]. Until 2011, chicken anemia virus (CAV) was the only Gyrovirus member identified. From that moment, several novel gyroviruses were characterized in humans and birds [52-54]. Partial genome of CAV was found in the present study. This virus is responsible for economic losses in poultry industry since it has tropism for bone marrow-derived cells, causing anemia and immunosuppression [55]. It was thought that CAV has chickens as only natural host, although antibodies in Coturnix japonica were detected [56]. Thus far, no other domestic or wild bird was associated to this virus [57]. This is the first report to characterize the presence of CAV in wild birds. By maximum-likelihood and neighbor joining analyses, this novel isolate is most closely related to Brazilian isolates (personal observation). CAV is also reported in mouse, dog, cat, and human faeces, besides human blood [58,59]. However, pathogenesis was not determined for these species. Nearly complete genome of avian gyrovirus 2 (AGV2) was obtained. AGV2 was the second member of Gyrovirus described and was first found in sick chickens in the south region of Brazil [60]. It was also identified in chickens with neurologic symptoms in South Africa and involved in infections of healthy people and transplant and HIV-positive patients [61,62]. This is a virus with worldwide distribution and with potential pathogenic importance [61-63]. The present isolate belongs to group A and is more closely related to the Chinese isolate HLJ1508 than Brazilian isolates, suggesting different origins of AGV2 in Brazil (Figure 7) [63]. AGV2 was also identified in chickens, ferrets and humans [60,62,64]. This is the first description of this virus in wild birds. Nearly complete genome of gyrovirus GyV3 was obtained (Figure 6A). This species was described in humans in Chile, in chickens in China and in ferrets in Hungary, all showing signs of disease [52,64,65]. This is the first report of GyV3 in Brazil. The present isolate is phylogenetically closer to the Chinese isolate SDAU-1. A possible association is supposed between this virus and the transmissible viral proventriculitis (TVP) disease in chickens and 
diarrhea in children $[52,64,65]$. Additionally, ORF1 nucleotide sequence of contigs NODE 117, NODE 986 and NODE 1090 showed low identity to the closest viral species, giant panda anellovirus (GpAV), and between them. Therefore, three probable novel anelloviruses species were identified in this study, the proposed brazilian bird anellovirus type 1, brazilian bird anellovirus type 2, and brazilian bird anellovirus type 3, respectively. Considering the expected genome size of the group, it is probably that contigs NODE 117 and NODE 986 sequences represents nearly complete genomes (Figure 6B). These viruses are related to a clade of unassigned members that includes GpAV, and feline anellovirus strain FelineAV621 [66-68] (Figure 8). Since the contigs were obtained from pool1, all the anellovirus sequences have origin in a neognath bird.

Circoviridade was other viral family identified. The cut-off criterium for species demarcation in this family is $80 \%$ complete genome nucleotide sequence identity [44]. BFDV was identified in pool 1, with $A$. aestiva as probable host. BFDV is responsible for a common and fatal disease in psittacines characterized by symmetric and progressive feather dystrophy and beak deformities, with no commercial vaccine available $[69,70]$. In its acute state, it has a high mortality rate, and, in chronic form, birds usually die by secondary infection caused by viral immunosuppression [71]. It has worldwide distribution and is a threat to psittacine conservation and the market of wild birds, especially in Brazil, that harbors great biodiversity of these species [72]. Although the first report in the country dates to 1998, there is few epidemiologic information about circulating viral isolates in the country [73]. Besides, BFDV shows higher mutation rate than other DNA viruses, which rate is similar to RNA viruses. In addition, the recombination is frequent in this species, specifically in the $3^{\prime}$ end of the Cap gene and intergenic region. These mechanisms can explain host diversity and help to support that probably all psittacines can be infected [33]. The present study describes the first complete genome sequence of a BFDV isolate in Brazil. Phylogenetic analysis, performed by clusters using CD-HIT 4.8.1 [74], indicates that the Brazilian isolate is closer to the Poland isolate, BFDV-U_PL-543_2008, with no recombination event detected using RDP4 v.4.96 (Figure 10) [75]. Additionally, a point mutation was observed that changes a cytosine $(\mathrm{C})$ to a thymine $(\mathrm{T})$ and drives an ochre stop codon, producing a truncated capsid protein. Further investigation is necessary to explain the evolutionary history of BFDV in A. aestiva host.

Noroviruses-like sequences were found. Members of genus Norovirus are especially known to cause gastroenteritis in humans and other hosts. Based on VP1 amino acid sequence, this genus is divided in seven genogroups [76]. GI, GII, GIV, and GVI infect humans, with just GI infecting solely this group [77,78]. In GII, there are viruses able to infect pigs [79]. In GIV, dogs, cats, and lions are hosts [80-82]. In GVI, dogs are infected [83]. Another genogroups, GIII, GV, and GVII, are thus far only associated to non-human animals, specifically ruminants (bovines and ovines), murines, and dogs, respectively [84]. Identification of noroviruses in animals raises concern about their zoonotic potential. However, cross-transmission between animals and humans has not been documented. Some evidences support human norovirus (HuNoV) infection in dogs based on the ability of virus attachment to the histo-blood group antigens (HBGAs) receptor and the presence of HuNoV-specific antibodies in these animals, although it was not assigned any clinical disease. Recently, HuNoV GII was identified in wild birds, raising the possibility of these animals being involved in virus transmission [31]. Contigs of small length in pool 1 showed identity to norovirus GII and norovirus GI (Figure 9). However, due to their small size, we could not confirm which viral genogroups were present in our samples. This is the second report of putative noroviruses in birds documented, suggesting these animals as potential reservoirs [31].

Contigs with nearly complete or complete genome sequences from three putative novel species of the family Parvoviridae were obtained. Two of them belongs to Chapparvovirus, a novel genus but not recognized by ICTV, so far. The first species of this group identified, Eidolon helvum parvovirus 2 (EhPV-2), was found in throat swabs of Eidolon helvum fruit bats in Africa in 2013, but the genus was proposed just in 2017 with Porcine parvovirus 7 (PPV7) identification in lung tissues of pigs in China $[85,86]$. Chapparvoviruses were also found in turkey, rat, Tasmanian devil, chicken, red-crowned 
crane, and mice faeces, rectal swab of pigs, in grey partridges, in Desmodus rotundus kidneys, and in faeces of animals of the present study [87-94]. Screening whole-genome shotgun (WGS) sequences assemblies, chapparvovirus endogenous viral elements (EVE) were identified in vertebrates and more recently in invertebrates [94,95]. This shows that Chapparvovirus has a wide range of host species and supports that vertebrate parvoviruses are not monophyletic as was commonly thought. Besides, this genus includes potential pathogens such as the mouse kidney parvovirus (MKPV), which was associated to chronic nephropathy, raising concern about the involvement of other chapparvoviruses in diseases [96]. NS1 amino acid sequence identity is used as demarcation criteria for genus and species in Parvoviridae family, with $30 \%$ identity as threshold to novel genus and $95.0 \%$ to species [44]. Thus, in the present study, contigs k119 15398 and k119 1463 represent sequences from putative novel viral species, with the proposed names for psittacara leucophthalmus chapparvovirus and avian chapparvovirus, respectively (Figure 11). Contig k119 1463 was found in pool 1, therefore it is associated to a neognath bird host. Contig k119 15398 was identified in pool 2 and, by phylogenetics analyses, was grouped more closely to k119 1463, suggesting that a bird host, probably P. leucophthalmus, harbors this species. Basal position of bird infecting chapparvoviruses can mean that a possible transmission between vertebrates and arthropods occurred initially in this group. The other putative novel parvovirus, the avian adeno-associated virus isolate BR_DF species (contig k119 1997), identified in pool 1, belongs to Dependoparvovirus genus, that includes viruses that infect vertebrates, but replication in the cell usually depends on another virus, called helper, commonly adenoviruses, herpesviruses or papillomaviruses [97]. In the absence of the helper virus, the cell is nonpermissive and latent infection is established with viral genome integration. Generally, dependoparvoviruses are not pathogenic and are used as vectors for gene therapy (Figure 12) [98]. This novel species is closer to adeno-associated viruses of birds supporting that a neognath bird is the host. Also, some contigs closely related to viral sequences of Densovirinae subfamily were obtained due to the feeding habits of these animals.

CRESS viruses are a group of circular ssDNA viruses with a common origin that encode a replication initiator protein (rep). Smacoviridae is one of the new families of the group that was recognized by ICTV in 2018 and were thought until recently to have animals as possible hosts since all isolates were identified in faeces or in abdominal of dragonflies by metagenomics analyses [37]. However, CRISPR analysis of Candidatus Methanomassiliicoccus intestinalis identified smacovirus originated sequences, which suggests that the host of smacoviruses are most likely archaea [99]. Analyses considering amber codon usage also support this hypothesis. Species and genus criteria demarcation of Smacoviridae are based on genome-wide and rep amino acid sequences with cut-off of $77.0 \%$ and $40.0 \%$, respectively. The low nearly complete genome sequence identity of contig NODE 726 with the closest smacovirus identified (67.3\%), Lemur associated porprismacovirus 1 isolate SF5 suggests the presence of a novel species in pool 1, which belongs to the Porprismacovirus genus also analyzing pairwise amino acid identity of rep $(97.2 \%)$, the proposed avian associated porprismacovirus, that has a neognath bird as probable host (Figures 13 and 14).

Brazilian fauna has wide diversity, but the animal virome is little explored. The present study was able to identify known animal adenoviruses, anelloviruses, and circovirus. Also, novel putative species of adenovirus, anellovirus, parvovirus, and smacovirus were found. Most sequences obtained belong to non-enveloped ssDNA viruses with small genome (Anelloviridae, Circoviridae, and Parvoviridae). This is in accordance to other metagenomic investigations of faecal viromes [100-102]. Additionally, high-throughput sequencing using Illumina HiSeq 2500 platform with $100 \mathrm{nt}$ paired-end allowed the identification of not only complete or nearly complete small genomes but also relatively bigger genomes, as observed for Adenoviridade. Some genomes were obtained in singles contigs. However, regarding to RNA viruses, only calicivirus-like sequences were detected. This viral diversity was characterized despite of the small number of animals sampled and shows how wild animals have a complexity and little-known viral microbiome. Other studies support this scenario where small sample sizes where applied, as the 201 CRESS DNA viruses isolates found associated to faeces of 
two capybaras (Hydrochoerus hydrochaeris) [100] and the potentially novel virus genomes described in 10 specimens of fur seals in Brazil (Arctocephalus sp.) [102].

Although the nucleotide sequences reported in this study do not comprise full genomes, this initial characterization contributes to the knowledge of the viral populations that occur in wild animals from South America and has identified potential novel viruses that may be of interest for future studies. This is the first study to use high-throughput sequencing to explore the viral diversity of southern hemisphere wild animals. The findings presented here are expected to help to understand how viral infections in wild animals may impact the health of birds' population and its potential as sources of viruses which may potentially infect other animal species.

Supplementary Materials: The following are available online at http://www.mdpi.com/1999-4915/11/9/803/s1, Table S1: Accession numbers pool1; Table S2: Accession numbers pool2; Table S3: Sequences used in phylogenetic trees.

Author Contributions: Conceived and designed the experiments: F.S.C. and M.A.D. Performed the experiments: C.R.B., F.S.C. and M.A.D. Analyzed the data: F.S.C., F.L.M., J.M.F.S., M.A.D., and T.N. Contributed reagents/materials/analysis tools: B.M.R., D.S.T., and T.N. Contributed to the writing of the manuscript: B.M.R., C.R.B., D.S.T., F.S.C., F.L.M., J.S.M.R., M.A.D., and T.N.

Funding: This work was supported by grants from Fundação de Apoio a Pesquisa (FAP/DF grant numbers 193.001532/2016 and 0193.000416/2016) and Conselho Nacional de Desenvolvimento Científico e Tecnológico (CNPq grant number 407908/2013-7). The funders had no role in the study design, data collection and analysis, the decision to publish, nor in the preparation of the manuscript.

Acknowledgments: The authors thank Veterinary Hospital at UnB for providing the samples of animals.

Conflicts of Interest: The authors declare no conflict of interest.

\section{References}

1. Strassburg, B.; Brooks, T.; Feltran-Barbieri, R.; Iribarrem, A.; Crouzeilles, R.; Loyola, R.; Latawiec, A.; Oliveira Filho, F.J.; De Mattos Scaramuzza, C.; Scarano, F.; et al. Moment of truth for the Cerrado hotspot. Nat. Ecol. Evol. 2017, 1, 1-3. [CrossRef] [PubMed]

2. De Figueiredo, M.L.G.; Figueiredo, L.T.M. Emerging alphaviruses in the Americas: Chikungunya and mayaro. Rev. Soc. Bras. Med. Trop. 2014, 47, 677-683. [CrossRef] [PubMed]

3. Silva, M.; Auguste, A.; Terzian, A.; Vedovello, D.; Riet-Correa, F.; Macário, V.; Mourão, M.P.G.; Ullmann, L.S.; Weaver, S.C.; Nogueira, M.L.; et al. Isolation and characterization of Madariaga Virus from a horse in Paraíba state, Brazil. Transbound. Emerg. Dis. 2015, 64, 990-993. [CrossRef] [PubMed]

4. Sakkas, H.; Bozidis, P.; Franks, A.; Papadopoulou, C. Oropouche fever: A review. Viruses 2018, $10,175$. [CrossRef] [PubMed]

5. Donalisio, M.R.; Freitas, A.R.R.; Zuben, A.P.B.V. Arboviruses emerging in Brazil: Challenges for clinic and implications for public health. Rev. Saude Publica 2017, 51, 30. [CrossRef] [PubMed]

6. Hotez, P. Neglected Tropical Diseases in the Anthropocene: The Cases of Zika, Ebola, and Other Infections. PLoS Negl. Trop. Dis. 2016, 10, e0004648. [CrossRef] [PubMed]

7. Gummow, B. Challenges posed by new and re-emerging infectious diseases in livestock production, wildlife and humans. Livest. Sci. 2010, 130, 41-46. [CrossRef]

8. Chomel, B.B.; Belotto, A.; Meslin, F.X. Wildlife, exotic pets, and emerging zoonoses. Emerg. Infect. Dis. 2007, 13, 6-11. [CrossRef] [PubMed]

9. Souza, M.J. Bacterial and parasitic zoonoses of exotic pets. Vet. Clin. N. Am. Exot. Anim. Pract. 2009, 12, 401-415. [CrossRef]

10. Jones, K.E.; Patel, N.G.; Levy, M.A.; Storeygard, A.; Balk, D.; Gittleman, J.L.; Daszak, P. Global trends in emerging infectious diseases. Nature 2008, 451, 990-993. [CrossRef]

11. Carroll, D.; Daszak, P.; Wolfe, N.D.; Gao, G.F.; Morel, C.M.; Morzaria, S.; Pablos-Méndez, A.; Tomori, O.; Mazet, J.A. The Global Virome Project. Science 2018, 359, 872-874. [CrossRef] [PubMed]

12. Stang, A.; Korn, K.; Wildner, O.; Uberla, K. Characterization of virus isolates by particle associated nucleic acid PCR. J. Clin. Microbiol. 2005, 43, 716-720. [CrossRef] [PubMed]

13. FastQC: A quality Control Tool for High Throughput Sequence Data. Available online: http://www. bioinformatics.babraham.ac.uk/projects/fastqc (accessed on 30 March 2019). 
14. Bushnell, B.; Rood, J.; Singer, E. BBMerge-Accurate paired shotgun read merging via overlap. PLoS ONE 2017, 12, e0185056. [CrossRef] [PubMed]

15. Li, D.; Liu, C.M.; Luo, R.; Sadakane, K.; Lam, T.W. MEGAHIT: An ultra-fast single-node solution for large and complex metagenomics assembly via succinct de Bruijn graph. Bioinform 2015, 15, 1674-1676. [CrossRef] [PubMed]

16. Bankevich, A.; Nurk, S.; Antipov, D.; Gurevich, A.; Dvorkin, M.; Kulikov, A.S.; Lesin, V.; Nikolenko, S.; Pham, S.; Prjibelski, A.; et al. SPAdes: A new genome assembly algorithm and its applications to single-cell sequencing. J. Comput. Biol. 2012, 19, 455-477. [CrossRef]

17. Tamura, K.; Stecher, G.; Peterson, D.; Filipski, A.; Kumar, S. MEGA7: Molecular evolutionary genetics analysis version 7.0. Mol. Biol. Evol. 2013, 30, 2725-2729. [CrossRef]

18. Stamatakis, A. RAxML version 8: A tool for phylogenetic analysis and post-analysis of large phylogenies. Bioinformatics 2014, 30, 1312-1313. [CrossRef]

19. Nguyen, L.T.; Schmidt, H.A.; von Haeseler, A.; Minh, B.Q. IQ-TREE: A fast and effective stochastic algorithm for estimating maximum-likelihood phylogenies. Mol. Biol. Evol. 2014, 32, 268-274. [CrossRef]

20. Collar, N.; Boesman, P.; Sharpe, C.J. White-eyed Parakeet (Psittacara leucophthalmus). In Handbook of the Birds of the World Alive, 1st ed.; Del Hoyo, J., Elliott, A., Sargatal, J., Christie, D.A., de Juana, E., Eds.; Lynx Edicions: Barcelona, Spain, 2019.

21. Rising, J.; Jaramillo, A. Saffron Finch (Sicalis flaveola). In Handbook of the Birds of the World Alive, 1st ed.; del Hoyo, J., Elliott, A., Sargatal, J., Christie, D.A., de Juana, E., Eds.; Lynx Edicions: Barcelona, Spain, 2019.

22. Gardner, A.L. Order Didelphimorphia. In Mammal. Species of the World: A Taxonomic and Geographic Reference, 3rd ed.; Wilson, D.E., Reeder, D.M., Eds.; Johns Hopkins University Press: Baltimore, EUA, 2005.

23. Groves, C.P. Order primates. In Mammal. Species of the World: A Taxonomic and Geographic Reference, 3rd ed.; Wilson, D.E., Reeder, D.M., Eds.; Johns Hopkins University Press: Baltimore, EUA, 2005.

24. Favoretto, S.; Araújo, D.; Naylê, F.H.; Oliveira, D.; da Crus, N.G.; Mesquita, F.; Leal, F.; Machado, R.R.G.; Gaio, F.; Oliveira, W.F.; et al. Zika Virus in peridomestic neotropical primates, Northeast Brazil. Ecohealth 2019, 16, 61-69. [CrossRef]

25. Wozencraft, W.C. Order Carnivora. In Mammal. Species of the World: A Taxonomic and Geographic Reference, 3rd ed.; Wilson, D.E., Reeder, D.M., Eds.; Johns Hopkins University Press: Baltimore, EUA, 2005.

26. King, A.M.Q.; Adams, M.J.; Carstens, E.B.; Lefkowitz, E.J. Family Adenoviridae. In Virus Taxonomy: 9th Report of the International Committee on Taxonomy of Viruses; Elsevier: Oxford, UK, 2011.

27. Binder, A.M.; Biggs, H.M.; Haynes, A.K.; Chommanard, C.; Lu, X.; Erdman, D.D.; Watson, J.T.; Gerber, S.I. Human adenovirus surveillance-United States, 2003-2016. MMWR Morb. Mortal. Wkly. Rep. 2017, 66, 1039-1042. [CrossRef]

28. Li, Y.; Ge, X.; Zhang, H.; Zhou, P.; Zhu, Y.; Zhang, Y.; Yuan, J.; Wang, L.F.; Shi, Z. Host range, prevalence, and genetic diversity of adenoviruses in bats. J. Virol. 2010, 84, 3889-3897. [CrossRef] [PubMed]

29. Souza, W.; Fumagalli, M.; Araujo, J.; Sabino-Santos Jr, G.; Felipe, G.M.M.; Romeiro, M.; Modha, S.; Nardi, M.S.; Queiroz, L.; Durigon, E.L.; et al. Discovery of novel anelloviruses in small mammals expands the host range and diversity of the Anelloviridae. Virology 2017, 514, 9-17. [CrossRef] [PubMed]

30. Kemenesi, G.; Gellért, Á.; Dallos, B.; Görföl, T.; Boldogh, S.; Estók, P.; Marton, S.; Oldal, M.; Martella, V.; Bányai, K.; et al. Sequencing and molecular modeling identifies candidate members of Caliciviridae family in bats. Infect. Genet. Evol. 2016, 41, 227-232. [CrossRef] [PubMed]

31. Summa, M.; Henttonen, H.; Maunula, L. Human noroviruses in the faeces of wild birds and rodents-new potential transmission routes. Zoonoses Public Health 2018, 65, 512-518. [CrossRef] [PubMed]

32. Rosario, K.; Breitbart, M.; Harrach, B.; Segalés, J.; Delwart, E.; Biagini, P.; Varsani, A. Revisiting the taxonomy of the family circoviridae: Establishment of the genus cyclovirus and removal of the genus gyrovirus. Arch. Virol. 2017, 162, 1447-1463. [CrossRef] [PubMed]

33. Sarker, S.; Ghorashi, S.A.; Forwood, J.K.; Bent, S.J.; Peters, A.; Raidal, S.R. Phylogeny of beak and feather disease virus in cockatoos demonstrates host generalism and multiple-variant infections within Psittaciformes. Virology 2014, 460, 72-82. [CrossRef] [PubMed]

34. Geldenhuys, M.; Mortlock, M.; Weyer, J.; Bezuidt, O.; Seamark, E.C.J.; Kearney, T.; Gleasner, C.; Erkkila, T.H.; Cui, H.; Markotter, W. A metagenomic viral discovery approach identifies potential zoonotic and novel mammalian viruses in Neoromicia bats within South Africa. PLoS ONE 2018, 13, e0194527. [CrossRef] 
35. François, S.; Filloux, D.; Roumagnac, P.; Bigot, D.; Gayral, P.; Martin, D.P.; Froissart, R.; Ogliastro, M. Discovery of parvovirus-related-sequences in an unexpected broad range of animals. Sci. Rep. 2016, 6, 30880. [CrossRef]

36. Cotmore, S.F.; Agbandje-McKenna, M.; Chiorini, J.A.; Mukha, D.V.; Pintel, D.J.; Qiu, J.; Soderlund-Venermo, M.; Tattersall, P.; Tijssen, P.; Gatherer, D.; et al. The family Parvoviridae. Arch. Virol. 2014, 159, 1239. [CrossRef]

37. Varsani, A.; Krupovic, M. Smacoviridae: A new family of animal-associated single-stranded DNA viruses. Arch. Virol. 2018, 163, 2005-2015. [CrossRef]

38. Chan, J.F.-W.; To, K.K.-W.; Chen, H.; Yuen, K.-Y. Cross-species transmission and emergence of novel viruses from birds. Curr. Opin. Virol. 2015, 10, 63-69. [CrossRef] [PubMed]

39. Bodewes, R. Novel viruses in birds: Flying through the roof or is a cage needed? Vet. J. 2018, $233,55-62$. [CrossRef] [PubMed]

40. Donaldson, E.F.; Haskew, A.N.; Gates, J.E.; Huynh, J.; Moore, C.J.; Frieman, M.B. Metagenomic analysis of the viromes of three North American bat species: viral diversity among different bat species that share a common habitat. J. Virol. 2010, 84, 13004-13018. [CrossRef] [PubMed]

41. Mishra, N.; Fagbo, S.F.; Alagaili, A.n.; Nitido, A.; Williams, S.H.; Lee, J.B.; Durosinlorun, A.; Garcia, J.E.; Jain, K.; Kapoor, V.; et al. A viral metagenomic survey identifies known and novel mammalian viruses in bats from Saudi Arabia. PLoS ONE 2019, 14, e0214227. [CrossRef] [PubMed]

42. Conceição-Neto, N.; Godinho, R.; Álvares, F.; Yinda, K.; Deboutte, W.; Zeller, M.; Laenen, L.; Heylen, E.; Roque, S.; Petrucci-Fonseca, F.; et al. Viral gut metagenomics of sympatric wild and domestic canids, and monitoring of viruses: Insights from an endangered wolf population. Ecol. Evol. 2017, 7, 4135-4146. [CrossRef] [PubMed]

43. Wu, Z.; Lu, L.; Du, J.; Yang, L.; Ren, X.; Liu, B.; Jiang, J.; Yang, J.; Dong, J.; Sun, L.; et al. Comparative analysis of rodent and small mammal viromes to better understand the wildlife origin of emerging infectious diseases. Microbiome 2018, 6, 178. [CrossRef] [PubMed]

44. International Committee on Taxonomy of Viruses Virus Taxonomy: 2018b Release. Available online: talk. ictvonline:ictv-reports/ictv_9th_report (accessed on 18 August 2019).

45. To, K.K.W.; Tse, H.; Chan, W.-M.; Choi, G.K.Y.; Anna, J.X.Z.; Sridhar, S.; Wong, S.; Chan, J.; Chan, A.S.F.; Woo, P.C.Y.; et al. A novel psittacine adenovirus identified during an outbreak of avian chlamydiosis and human psittacosis: Zoonosis associated with virus-bacterium coinfection in birds. PLoS Negl. Trop. Dis. 2014, 8, e3318. [CrossRef]

46. Hess, M.; Blöcker, H.; Brandt, P. The complete nucleotide sequence of the egg drop syndrome Virus: An intermediate between Mastadenoviruses and Aviadenoviruses. Virology 1997, 238, 145-156. [CrossRef]

47. Schrenzel, M.; Oaks, J.L.; \& Rotstein, D.; Maalouf, G.; Snook, E.; Sandfort, C.; Rideout, B. Characterization of a new species of adenovirus in falcons. J. Clin. Microbiol. 2005, 43, 3402-3413. [CrossRef]

48. Schachner, A.; Matos, M.; Grafl, B.; Hess, M. Fowl adenovirus-induced diseases and strategies for their control-A review on the current global situation. Avian Pathol. 2017, 47, 111-126. [CrossRef]

49. Chiocca, S.; Kurzbauer, R.; Schaffner, G.; Baker, A.; Mautner, V.; Cotten, M. The complete DNA sequence and genomic organization of the avian adenovirus CELO. J. Virol. 1996, 70, 2939-2949. [PubMed]

50. Phan, T.G.; Li, L.; O’Ryan, M.G.; Cortes, H.; Mamani, N.; Bonkoungou, I.J.O.; Wang, C.; Leutenegger, C.M.; Delwartcorresponding, E. A third gyrovirus species in human faeces. J. Gen. Virol. 2012, 93, 1356-1361. [CrossRef] [PubMed]

51. Smuts, H. Novel gyroviruses, including chicken anaemia virus, in clinical and chicken samples from South Africa. Adv. Virol. 2014, 2014. [CrossRef] [PubMed]

52. Li, L.; Pesavento, P.A.; Gaynor, A.M.; Duerr, R.S.; Phan, T.G.; Zhang, W.; Deng, X.; Delwart, E. A gyrovirus infecting a sea bird. Arch. Virol. 2015, 160, 2105-2109. [CrossRef] [PubMed]

53. Shuai, Y.; Tianbei, T.; Xiang, G.; Chunyan, H.; Nana, Y.; Aijing, L.; Honglei, G.; Yulong, G.; Hongyu, C.; Changjun, L.; et al. Molecular epidemiology of chicken anaemia virus in sick chickens in China from 2014 to 2015. PLoS ONE 2019, 14, e0210696. [CrossRef]

54. Farkas, T.; Maeda, K.; Sugiura, H.; Kai, K.; Hirai, K.; Otsuki, K.; Hayashi, T. A serological survey of chickens, Japanese quail, pigeons, ducks and crows for antibodies to chicken anaemia virus (CAV) in Japan. Avian Pathol. 1998, 27, 316-320. [CrossRef] [PubMed] 
55. Tang, M.-B.; Chang, H.-M.; Wu, W.-C.; Chou, Y.-C.; Yu, C.-P. First detection of chicken anemia virus and norovirus genogrupo II in stoll of children with acute gastroenteritis in Taiwan. Southeast Asian J. Trop. Med. Public Health 2016, 47, 416-423.

56. Lichun, F.; Yang, L.; Yixin, W.; Jiayuan, F.; Shuai, C.; Xiaohan, L.; Shuang, C.; Peng, Z. Genetic analysis of two chicken infectious anemia virus variants-related gyrovirus in stray mice and dogs: The first report in China, 2015. Biomed. Res. Int. 2017, 2017. [CrossRef]

57. Xinheng, Z.; Yuanjia, L.; Jun, J.; Feng, C.; Baoli, S.; Chunyi, X.; Jingyun, M.; Yingzuo, B.; Qingmei, X. Identification of a chicken anemia virus variant-related Gyrovirus in stray cats in China, 2012. Biomed. Res. Int. 2012, 2014. [CrossRef]

58. Maggi, F.; Macera, L.; Focosi, D.; Vatteroni, M.L.; Boggi, U.; Antonelli, G.; Eloit, M.; Pistello, M. Human gyrovirus DNA in human blood, Italy. Emerg. Infect. Dis. 2012, 18, 956-959. [CrossRef]

59. Chu, D.K.; Poon, L.L.; Chiu, S.S.; Chan, K.H.; Ng, E.M.; Bauer, I.; Cheung, T.K.; Ng, I.H.; Guan, Y.; Wang, D.; et al. Characterization of a novel gyrovirus in human stool and chicken meat. J. Clin. Virol. 2012, 55, 209-213. [CrossRef] [PubMed]

60. Rijsewijk, F.A.; Dos Santos, H.F.; Teixeira, T.F.; Cibulski, S.P.; Varela, A.P.; Dezen, D.; Franco, A.C.; Roehe, P.M. Discovery of a genome of a distant relative of chicken anemia virus reveals a new member of the genus Gyrovirus. Arch. Virol. 2011, 156, 1097-1100. [CrossRef] [PubMed]

61. Abolnik, C.; Wandrag, D. Avian gyrovirus 2 and avirulent Newcastle disease virus coinfection in a chicken flock with neurologic symptoms and high Mortalities. Avian Dis. 2014, 58, 90-94. [CrossRef] [PubMed]

62. Biagini, P.; Bedarida, S.; Touinssi, M.; Galicher, V.; Philippe de, M. Human gyrovirus in healthy blood donors, France. Emerg. Infect. Dis. 2013, 19, 1014-1015. [CrossRef] [PubMed]

63. Yao, S.; Gao, X.; Tuo, T.; Han, C.; Gao, Y.; Qi, X.; Zhang, Y.; Liu, C.; Gao, H.; Wang, Y.; et al. Novel characteristics of the avian gyrovirus 2 genome. Sci. Rep. 2017, 7, 41068. [CrossRef] [PubMed]

64. Fehér, E.; Pazár, P.; Mihalov-Kovács, E.; Farkas, S.; Lengyel, G.; Jakab, F.; Martella, V.; Banyai, K. Molecular detection and characterization of human gyroviruses identified in the ferret fecal virome. Arch. Virol. 2014, 159, 3401-3406. [CrossRef]

65. Li, G.; Yuan, S.; He, M.; Zhao, M.; Hao, X.; Song, M.; Zhang, L.; Qiao, C.; Huang, L.; Zhang, L.; et al. Emergence of gyrovirus 3 in commercial broiler chickens with transmissible viral proventriculitis. Transbound. Emerg. Dis. 2018, 65, 1170-1174. [CrossRef] [PubMed]

66. Zhang, W.; Yang, S.; Shan, T.; Hou, R.; Liu, Z.; Li, W.; Guo, L.; Wang, Y.; Chen, P.; Wang, X.; et al. Virome comparisons in wild-diseased and healthy captive giant pandas. Microbiome 2017, 5, 90. [CrossRef] [PubMed]

67. Zhang, W.; Wang, H.; Wang, Y.; Liu, Z.; Li, J.; Guo, L.; Yang, S.; Shen, Q.; Zhao, X.; Cui, L.; et al. Identification and genomic characterization of a novel species of feline anellovirus. Virol. J. 2016, 13, 146. [CrossRef] [PubMed]

68. Okamoto, H.; Takahashi, M.; Nishizawa, T.; Tawara, A.; Fukai, K.; Muramatsu, U.; Naito, Y.; Yoshikawa, A.J. Genomic characterization of TT viruses (TTVs) in pigs, cats and dogs and their relatedness with species-specific TTVs in primates and tupaias. Gen. Virol. 2002, 83, 1291-1297. [CrossRef]

69. Pass, D.A.; Perry, R.A. The pathology of psittacine beak and feather disease. Aust. Vet. J. 1984, 61, 69-74. [CrossRef] [PubMed]

70. Ritchie, B.W.; Niagro, F.D.; Lukert, P.D.; Steffens, W.L.; Latimer, K.S. Characterization of a new virus from cockatoos with psittacine beak and feather disease. Virology 1989, 171, 83-88. [CrossRef]

71. Todd, D. Circoviruses: Immunosuppressive threats to avian species: A review. Avian Pathol. 2000, $29,373-394$. [CrossRef] [PubMed]

72. Ortiz-Catedral, L.; McInnes, K.; Hauber, M.E.; Brunton, D.H. First report of beak and feather disease virus (BFDV) in wild Red-fronted Parakeets (Cyanoramphus novaezelandiae) in New Zealand. Emu 2009, 109, 244-247. [CrossRef]

73. Werther, K.; Raso, T.F.; Durigon, E.L.; Latimer, K.S.; Campagnoli, R.P. Description of the first case of psittacine beak and feather disease in Brazil. In Proceedings of the International Virtual Conferences in Veterinary Medicine: Diseases of Psittacine Birds, Athens, GA, USA, 15-30 June 1998.

74. Weizhong, L.; Adam, G. Cd-hit: A fast program for clustering and comparing large sets of protein or nucleotide sequences. Bioinformatics 2006, 22, 1658-1659. [CrossRef]

75. Martin, D.P.; Murrell, B.; Golden, M.; Khoosal, A.; Muhire, B. RDP4: Detection and analysis of recombination patterns in virus genomes. Virus Evol. 2015, 1. [CrossRef] [PubMed] 
76. Todd, K.; Tripp, R. Human Norovirus: Experimental Models of Infection. Viruses 2019, 11, 151. [CrossRef]

77. Siebenga, J.J.; Vennema, H.; Zheng, D.P.; Vinje', J.; Lee, B.E.; Pang, X.L.; Ho, E.C.; Lim, W.; Choudekar, A.; Broor, S.; et al. Norovirus illness is a global problem: Emergence and spread of norovirus GII.4 variants. J. Infect. Dis. 2009, 200, 802-812. [CrossRef]

78. Mesquita, J.R.; Costantini, V.P.; Cannon, J.L.; Lin, S.C.; Nascimento, M.S.; Vinjé, J. Presence of antibodies against genogroup VI norovirus in humans. Virol. J. 2013, 10, 176. [CrossRef]

79. Wang, Q.H.; Han, M.; Cheetham, S.; Souza, M.; Funk, J.; Saif, L. Porcine noroviruses related to human noroviruses. Emerg. Infect. Dis. 2005, 11, 1874-1881. [CrossRef]

80. Martella, V.; Lorusso, E.; Decaro, N.; Elia, G.; Radogna, A.; D'Abramo, M.; Buonavoglia, C. Detection and molecular characterization of a canine norovirus. Emerg. Infect. Dis. 2008, 14, 1306-1308. [CrossRef] [PubMed]

81. Pinto, P.; Wang, Q.; Chen, N.; Dubovi, E.; Daniels, J.B.; Millward, L.M.; Buonavoglia, C.; Martella, V.; Saif, L. Discovery and Genomic Characterization of noroviruses from a gastroenteritis outbreak in domestic cats in the US. PLoS ONE 2012, 7, e32739. [CrossRef] [PubMed]

82. Martella, V.; Campolo, M.; Lorusso, E.; Cavicchio, P.; Camero, M.; Bellacicco, A.L.; Decaro, N.; Elia, G.; Greco, G.; Corrente, M.; et al. Norovirus in captive lion cub (Panthera leo). Emerg. Infect. Dis. 2007, 13, 1071-1073. [CrossRef]

83. Caddy, S.; Emmott, E.; El-Attar, L.; Mitchell, J.; Rougemont, A.; Brownlie, J.; Goodfellow, I. Serological evidence for multiple strains of canine norovirus in the UK dog population. PLoS ONE 2013, 8, e81596. [CrossRef] [PubMed]

84. Zheng, D.P.; Ando, T.; Fankhauser, R.L.; Beard, R.S.; Glass, R.I.; Monroe, S.S. Norovirus classification and proposed strain nomenclature. Virology 2006, 346, 312-323. [CrossRef] [PubMed]

85. Baker, K.S.; Leggett, R.M.; Bexfield, N.H.; Alston, M.; Daly, G.; Todd, S.; Tachedjian, M.; Holmes, C.; Crameri, S.; Wang, L.F.; et al. Metagenomic study of the viruses of African straw-coloured fruit bats: Detection of a chiropteran poxvirus and isolation of a novel adenovirus. Virology 2013, 441, 95-106. [CrossRef] [PubMed]

86. Chen, R.J.; Lai, T.T.; Chen, Q.Y.; Wu, X.M.; Che, Y.L.; Yan, S.; Wang, C.Y.; Wang, L.B.; Zhou, L.J. Classification tree method for determining factors that affecting hatchability in chukar partridge (Alectoris chukar) eggs. Kafkas Univ. Vet. Fak. Derg 2018, 24, 473-477. [CrossRef]

87. Reuter, G.; Boros, Á.; Delwart, E.; Pankovics, P. Novel circular single-stranded DNA virus from turkey faeces. Arch. Virol. 2014, 159, 2161-2164. [CrossRef]

88. Yang, S.; Liu, Z.; Wang, Y.; Li, W.; Fu, X.; Lin, Y.; Shen, Q.; Wang, X.; Wang, H.; Zhang, W. A novel rodent Chapparvovirus in feces of wild rats. Virol. J. 2016, 13, 133. [CrossRef]

89. Chong, R.; Shi, M.; Grueber, C.; Holmes, E.; Hogg, C.; Belov, K.; Barrs, V. Characterisation of the faecal virome of captive and wild Tasmanian devils using virus-like particles metagenomics and meta-transcriptomics. bioRxivorg 2018, 443457. [CrossRef]

90. Lima, D.; Cibulski, S.; Tochetto, C.; Varela, A.P.M.; Finkler, F.; Teixeira, T.F.; Loiko, M.; Cerva, C.; Junqueira, D.; Mayer, F.; et al. The intestinal virome of malabsorption syndrome-affected and unaffected broilers through shotgun metagenomics. Virus Res. 2018, 261, 9-20. [CrossRef] [PubMed]

91. Wang, Y.; Yang, S.; Liu, D.; Zhou, C.; Li, W.; Lin, Y.; Wang, X.; Shen, Q.; Wang, H.; Li, C.; et al. The fecal virome of red-crowned cranes. Arch. Virol. 2018, 164, 1-14. [CrossRef] [PubMed]

92. Williams, S.H.; Che, X.; Garcia, J.A.; Klena, J.D.; Lee, B.; Muller, D.; Ulrich, W.; Corrigan, R.M.; Nichol, S.; Jain, K.; et al. Viral Diversity of House Mice in New York City. MBio 2018, 9, e01354-17. [CrossRef] [PubMed]

93. Palinski, R.M.; Mitra, N.; Hause, B.M. Discovery of a novel Parvovirinae virus, porcine parvovirus 7, by metagenomic sequencing of porcine rectal swabs. Virus Genes 2016, 52, 564-567. [CrossRef] [PubMed]

94. Souza, W.; Romeiro, M.; Fumagalli, M.; Modha, S.; Araujo, J.; Queiroz, L.; Durigon, E.L.; Figueiredo, L.T.M.; Murcia, P.; Gifford, R. Chapparvoviruses occur in at least three vertebrate classes and have a broad biogeographic distribution. J. Gen. Virol. 2017, 98, 225-229. [CrossRef] [PubMed]

95. Gifford, R.; Agbandje-McKenna, M.; Souza, W.; Pénzes, J. An ancient lineage of highly divergent parvoviruses infects both vertebrate and invertebrate hosts. bioRxivorg 2019, 11, 525. [CrossRef]

96. Roediger, B.; Lee, Q.; Tikoo, S.; Cobbin, J.; Henderson, J.; Jormakka, M.; O’Rourke, M.; Padula, M.; Pinello, N.; Henry, M.; et al. An atypical parvovirus drives chronic tubulointerstitial nephropathy and kidney fibrosis. Cell 2018, 175, 530-543. [CrossRef] 
97. Strauss, J.H.; Strauss, E.G. Viruses and Human Disease, 2nd ed.; Academic Press: Cambridge, MA, USA, 2008; pp. 261-323.

98. Berns, K.I.; Muzyczka, N. AAV: An Overview of unanswered questions. Hum. Gene. Ther. 2017, 28, 308-313. [CrossRef]

99. Díez-Villaseñor, C.; Rodríguez-Valera, F. CRISPR analysis suggests that small circular single-stranded DNA smacoviruses infect Archaea instead of humans. Nat. Commun. 2018, 10, 294. [CrossRef]

100. Fontenele, R.S.; Lacorte, C.; Lamas, N.S.; Schmidlin, K.; Varsani, A.; Ribeiro, S.G. Single stranded DNA viruses associated with capybara faeces sampled in Brazil. Viruses 2019, 11, 710. [CrossRef]

101. Kluge, M.; Campos, F.; Tavares, M.; Amorim, D.; Pedone Valdez, F.; Giongo, A.; Roehe, P.; Franco, A. Metagenomic survey of viral diversity obtained from feces of subantarctic and south american fur seals. PLoS ONE 2016, 11, e0151921. [CrossRef] [PubMed]

102. Karlsson, L.O.; Larsson, J.; Hayer, J.; Berg, M.; Jacobson, M. The intestinal eukaryotic virome in healthy and diarrhoeic neonatal piglets. PLoS ONE 2016, 11, e0151481. [CrossRef] [PubMed]

(C) 2019 by the authors. Licensee MDPI, Basel, Switzerland. This article is an open access article distributed under the terms and conditions of the Creative Commons Attribution (CC BY) license (http://creativecommons.org/licenses/by/4.0/). 